JOURNAL OF THE

AMERICAN MATHEMATICAL SOCIETY

Volume 11, Number 1, January 1998, Pages 1-39

S 0894-0347(98)00247-1

\title{
PHRAGMÉN-LINDELÖF PRINCIPLES ON ALGEBRAIC VARIETIES
}

\author{
R. MEISE, B. A. TAYLOR, AND D. VOGT
}

\section{INTRODUCTION}

From several results in recent years, starting with Hörmander's characterization of the constant coefficient partial differential equations $P(D) u=f$ that have a real analytic solution $u$ for every real analytic function $f$, it has become clear that certain properties of the partial differential operator $P(D)$ are equivalent to estimates of Phragmén-Lindelöf type for plurisubharmonic functions on the algebraic variety

$$
V=V(P):=\left\{z \in \mathbb{C}^{n}: P(z)=0\right\}
$$

(see Hörmander [15], Zampieri [32], Kaneko [16], [17], Braun, Meise and Vogt [7], [8], Braun [3], [4], Meise, Taylor and Vogt [19], [20], [21], [23], [25], Palamodov [30] and Momm [28]). There are several different such estimates that are of interest, corresponding to different properties of the operator, e.g. surjectivity, existence of a continuous linear right inverse, continuation of solutions. Their importance is that, in many cases, they seem to provide the only way to evaluate whether or not a given operator $P(D)$ has the given property.

In the present paper we study those sets of estimates which have been shown in [21] and [23] (see Palamodov [30] for the case of systems) to be equivalent to the operator $P(D): C^{\infty}(\Omega) \longrightarrow C^{\infty}(\Omega)$ having a continuous linear right inverse, $\Omega$ a convex open subset of $\mathbb{R}^{n}$. In the important special case $\Omega=\mathbb{R}^{n}$, the set of estimates is called $\operatorname{PL}\left(\mathbb{R}^{n}, \log \right)$ and can be stated as follows:

An algebraic variety $V$ in $\mathbb{C}^{n}$ satisfies the Phragmén-Lindelöf condition $\operatorname{PL}\left(\mathbb{R}^{n}, \log \right)$ if there is a number $A>0$ such that for each $\rho>0$ there is a constant $B=B(\rho)>0$ such that each plurisubharmonic function $u$ on $V$ satisfying (with $|\cdot|$ denoting the Euclidean norm)

(1) $u(z) \leq|\operatorname{Im} z|+O(\log (2+|z|))$ for all $z \in V$

and

(2) $u(z) \leq \rho|\operatorname{Im} z|$ for all $z \in V$

also satisfies

(3) $u(z) \leq A|\operatorname{Im} z|+B \log (2+|z|)$ for all $z \in V$.

If we replace $\log (2+|z|)$ in the above by a weight function $\omega(|z|)$ (see 2.1) we get the condition $\operatorname{PL}\left(\mathbb{R}^{n}, \omega\right)$. For a complex polynomial $P$ in $n$ variables the variety $V=V(P):=\left\{z \in \mathbb{C}^{n}: P(-z)=0\right\}$ satisfies the condition $\operatorname{PL}(\Omega, \omega)$ if and only if the differential operator $P(D)$ on the nonquasianalytic class $\mathcal{E}_{(\omega)}\left(\mathbb{R}^{n}\right)$

Received by the editors June 28, 1994 and, in revised form, July 13, 1994.

1991 Mathematics Subject Classification. Primary 32F05; Secondary 46 J99.

The second named author gratefully acknowledges the support of his research by the "Gesellschaft von Freunden und Förderern der Heinrich-Heine-Universität Düsseldorf". 
of $\omega$-ultradifferentiable functions of Beurling type admits a continuous linear right inverse (see [23]).

If we replace (1) above by $(1)^{\prime} u(z) \leq|z|$ for all $z \in V$

and if the polynomial $P$ is homogeneous, then we get the condition $\operatorname{HPL}\left(\mathbb{R}^{n}\right)$ which was studied by Hörmander [15], who was the first to investigate such conditions. Hörmander's condition is different from the one studied here. It characterizes the polynomials $P$ such that $P(D)$ is a surjective linear map on the real analytic functions. Thus, the zero sets of elliptic operators satisfy $\operatorname{HPL}\left(\mathbb{R}^{n}\right)$. On the other hand, it is a theorem of Grothendieck that elliptic operators never have a continuous linear right inverse on $C^{\infty}(\Omega)$ so their zero sets must fail $\operatorname{PL}\left(\mathbb{R}^{n}, \log \right)$. Another difference in the conditions is that, as proved by Hörmander, the validity of the condition $\operatorname{HPL}\left(\mathbb{R}^{n}\right)$ is not changed by lower degree perturbations of the polynomial while, as we show here, the condition $\operatorname{PL}\left(\mathbb{R}^{n}, \omega\right)$ may be.

In general, it is difficult to decide whether a given algebraic variety satisfies the condition $\operatorname{PL}\left(\mathbb{R}^{n}, \omega\right)$. Ideally, one would like to find geometric conditions on the variety that characterize $\operatorname{PL}\left(\mathbb{R}^{n}, \log \right)$. Our effort here to find such conditions consists of three main parts; the study of homogeneous varieties (Section 3), giving necessary conditions (Section 4), and giving sufficient conditions (Section 5). Necessary conditions allow us to check examples that fail $\operatorname{PL}\left(\mathbb{R}^{n}, \omega\right)$ while sufficient conditions are used to give positive examples.

In the study of this problem, it is useful to know equivalent formulations. For the case of homogeneous varieties, various characterizations are given in Theorem 3.3. In particular, for homogeneous varieties the exact relationship between $\operatorname{PL}\left(\mathbb{R}^{n}, \omega\right)$ and $\operatorname{HPL}\left(\mathbb{R}^{n}\right)$ can be given. We prove that a homogeneous variety $V_{h}$ satisfies $\operatorname{PL}\left(\mathbb{R}^{n}, \omega\right)$ if and only if it satisfies one of the following conditions:

(i) $V_{h}$ satisfies the local Phragmén-Lindelöf condition $\operatorname{HPL}\left(\mathbb{R}^{n}\right.$, loc $)$ - introduced by Hörmander [15] — at the origin.

(ii) $V_{h}$ satisfies $\operatorname{HPL}\left(\mathbb{R}^{n}\right.$, loc $)$ at all points $\xi \in V_{h} \cap \mathbb{R}^{n},|\xi|=1$, and the dimension condition (i.e. $\operatorname{dim}_{\mathbb{R}} W \cap \mathbb{R}^{n}=\operatorname{dim}_{\mathbb{C}} W$ for each irreducible component $W$ of $\left.V_{h}\right)$.

(iii) $V_{h}$ satisfies $\operatorname{HPL}\left(\mathbb{R}^{n}\right)$ and none of its irreducible components is elliptic.

These equivalences show that the condition is independent of $\omega$ for homogeneous varieties. They also allow easy verification of the validity or failure of $\operatorname{PL}\left(\mathbb{R}^{n}, \omega\right)$ for many homogeneous polynomials $P$. For example, the dimension condition of (ii) shows that a homogeneous variety must have a "maximal number of real zeros" in order that it satisfy the condition. This implies the result mentioned earlier that elliptic operators cannot have a continuous linear right inverse since the ellipticity condition is that $\{P(z)=0\} \cap \mathbb{R}^{n}=\{0\}$. Similarly, the symbols of operators like the principal part of a parabolic operator, e.g. $P\left(z_{1}, \ldots, z_{n}\right)=z_{1}^{2}+\cdots+z_{m}^{2}, \quad 1<$ $m \leq n$, have a real zero set of dimension less than $n-1$ and so also fail the dimension condition and hence, $\operatorname{PL}\left(\mathbb{R}^{n}, \omega\right)$. On the other hand, polynomials like $P\left(z_{1}, \ldots, z_{n}\right)=z_{1}^{3}+\cdots+z_{n}^{3}$, or indefinite nondegenerate quadratic forms such as $P\left(z_{1}, \ldots, z_{n}\right)=z_{1}^{2}-\left(z_{2}^{2}+\cdots+z_{n}^{2}\right)$ (wave operator), or $P\left(z_{1}, \ldots, z_{4}\right)=z_{1}^{2}+z_{2}^{2}-$ $z_{3}^{2}-z_{4}^{2}$ (ultrahyperbolic operator) do have a real zero set of maximal dimension and satisfy the dimension condition. Further, the zero varieties are nonsingular outside the origin and it is trivial that the local Phragmén-Lindelöf condition is satisfied at each real regular point of a variety. Therefore, by (ii), all the operators $P(D)$ for 
these examples do admit a continuous linear right inverse on $C^{\infty}\left(\mathbb{R}^{n}\right)$. Note that this is an existence proof. It does not give a "formula" for the solution operator whose existence is guaranteed by the validity of $\mathrm{PL}\left(\mathbb{R}^{n}, \omega\right)$. While this is classical for hyperbolic operators (or for operators with a good Cauchy problem, take the solution $u$ of $P(D) u=f$ with 0 Cauchy data), until recently we did not know how to do this for the other second-order operators, like the ultrahyperbolic operator. At the present time, we do not know how to construct a solution operator for many cases where we can prove that $\mathrm{PL}\left(\mathbb{R}^{n}, \log \right)$ holds. For example, the operator $\partial^{3} / \partial x_{1}^{3}+\cdots+\partial^{3} / \partial x_{n}^{3}$.

The case of nonhomogeneous varieties is not as well understood. For this case, using results and methods from [26] and [15], we prove a necessary condition that $\operatorname{PL}\left(\mathbb{R}^{n}, \omega\right)$ hold for $V$ is that its tangent cone at infinity, $V_{h}$ (which is homogeneous), also satisfies $\operatorname{PL}\left(\mathbb{R}^{n}, \omega\right)$ (Theorem 4.1). From the results about homogeneous varieties, this then shows that examples like $z_{1}^{2}+z_{2}^{2}+\lambda z_{3}=0$ fail $\operatorname{PL}\left(\mathbb{R}^{n}, \log \right)$ (e.g. parabolic operators, a result due to Cohoon [10], [11], or hypoelliptic operators, a result due to Vogt [31]). A further necessary condition for an irreducible $V$ to satisfy $\operatorname{PL}\left(\mathbb{R}^{n}, \omega\right)$ is the existence of a large subset $V_{0}$ of $V$ so that the distance from $z \in V$ to the tangent cone, $V_{h}$, is of the order of $\omega(z)$ or bounded in case $\omega(z)=\log (1+|z|)$, the case of $C^{\infty}\left(\mathbb{R}^{n}\right)$ (Proposition 4.8). For example, with the heat operator in one space variable, $z_{1}^{2}+i z_{2}$, the tangent cone at infinity is the $z_{2}$-axis, $\left\{\left(0, z_{2}\right)\right\}$, and the distance from a point with $z_{1}^{2}+i z_{2}=0$ to this line is $O(\sqrt{|z|})$. This variety satisfies $\operatorname{PL}\left(\mathbb{R}^{n}, \omega\right)$ when $\sqrt{|z|}=O(\omega(z))$ but not otherwise. However, an example given in 4.9 , namely $P\left(z_{1}, z_{2}, z_{3}\right)=\lambda z_{1}+z_{2} z_{3}$, shows that the distance, $\operatorname{dist}\left(z, V_{h}\right), z \in V$, can grow much faster than $\omega(z)$ if $V_{h}$ has singularities outside the origin. We show that this example satisfies $\operatorname{PL}\left(\mathbb{R}^{n}, \log \right)$ if and only if $\lambda$ is real. The example also indicates that it is difficult to treat a general polynomial as a perturbation of its principal part.

However, under the additional hypothesis that $V_{h}$ is a manifold outside the origin, it is shown in Section 5 that a perturbation result is valid. Namely, in this case $V$ satisfies $\operatorname{PL}\left(\mathbb{R}^{n}, \omega\right)$ if and only if $V_{h}$ satisfies $\operatorname{PL}\left(\mathbb{R}^{n}, \omega\right)$ and $\operatorname{dist}\left(z, V_{h}\right)=O(\omega(z))$ for $z \in V,|z| \rightarrow \infty$ (Theorem 5.6). To prove this, we use the main result from [26] on radial Phragmén-Lindelöf conditions to get a certain uniform estimate and then apply techniques of Hörmander [15]. This perturbation result gives many examples where $\operatorname{PL}\left(\mathbb{R}^{n}, \log \right)$ is verified, for example $P\left(z_{1}, z_{2}, z_{3}\right)=z_{1}^{3}+z_{2}^{3}+z_{3}^{3}+Q(z)$ where $Q$ is any polynomial of degree at most 2. See Corollary 5.8 for a general class of examples.

Some of the results stated above hold for arbitrary convex open subsets $\Omega$ of $\mathbb{R}^{n}$ and not only for $\mathbb{R}^{n}$.

The paper is organized as follows. In Section 2 we introduce the PhragménLindelöf conditions and derive equivalent formulations. Then we study the case of homogeneous varieties in Section 3. In Section 4 we derive necessary conditions for $\operatorname{PL}(\Omega, \omega)$ to hold on nonhomogeneous varieties. Sufficient conditions for nonhomogeneous varieties including the perturbation result are proved in Section 5. In Section 6 we introduce a further Phragmén-Lindelöf condition $\operatorname{PL}\{\Omega, \omega\}$ which by [23] is related with the existence of continuous linear right inverses on $\omega$-ultradifferentiable functions of Roumieu type. We show that the main results for $\operatorname{PL}(\Omega, \omega)$ carry over to $\operatorname{PL}\{\Omega, \omega\}$ by an easy reduction argument.

The authors thank R. W. Braun for many interesting and helpful discussions on the subject of the present article. 


\section{Definition of the Phragmén-Lindelöf COnditions}

In this section we introduce the Phragmén-Lindelöf conditions to be studied subsequently and we note some simple properties they satisfy. In order to state them, we have to introduce some notation.

Definition 2.1. Let $\omega:[0, \infty[\longrightarrow] 0, \infty$ [ be continuous and increasing and assume that it has the following properties:

$(\alpha) \omega(2 t)=O(\omega(t))$.

(в) $\int_{1}^{\infty} \frac{\omega(t)}{t^{2}} d t<\infty$

$(\gamma) \log t=O(\omega(t))$.

$(\delta) x \longmapsto \omega\left(e^{x}\right)$ is convex.

By abuse of notation $\omega: z \mapsto \omega(|z|), z \in \mathbb{C}^{n}$, will be called a weight function.

Note that each weight function satisfies $\omega(z)=o(|z|)$ by [18], p.731. Moreover, each weight function is plurisubharmonic (psh) in $\mathbb{C}^{n}$ in view of $2.1(\delta)$. Condition 2.1( $\beta$ ) implies the existence of the psh functions asserted by the following lemma, which is an immediate consequence of Braun and Meise [5], Prop.5.

Lemma 2.2. For each weight function $\omega$ on $\mathbb{C}^{n}$ and each $\varepsilon>0$ there exists a psh function $u_{\varepsilon}$ on $\mathbb{C}^{n}$ and $C_{\varepsilon}>0$ such that

$$
-C_{\varepsilon} \omega(z) \leq u_{\varepsilon}(z) \leq \varepsilon|\operatorname{Im} z|-\omega(z) \text { for all } z \in \mathbb{C}^{n} .
$$

There are several possible definitions of what is meant by a psh function on an analytic variety, see e.g. Fornaess and Narasimhan [12]. For our purposes it is convenient to have the largest possible class, the so-called weakly psh functions, which is defined in the following way:

Definition 2.3. Let $V$ be an analytic variety in an open subset $D$ of $\mathbb{C}^{n}$. A function $u: V \longrightarrow[-\infty, \infty$ [ is called psh on $V$ if it is locally bounded above and psh at the regular points $V_{\text {reg }} \subset V$. The values of $u$ at the singular points $V_{\text {sing }} \subset V$ are not important in our considerations. However, for the formulation of our results it is convenient in the sequel to assume that

$$
u(z)=\limsup _{\xi \in V_{\text {reg }}, \xi \rightarrow z} u(\xi) \quad \text { for all } z \in V_{\text {sing }} .
$$

By $\operatorname{PSH}(V)$ we denote the set of all psh functions on $V$ which satisfy this condition.

Definition 2.4. (a) For a convex open set $\emptyset \neq \Omega \subset \mathbb{R}^{n}$ we define

$$
\mathcal{K}(\Omega):=\{K \subset \Omega: K \quad \text { is convex and compact }\} .
$$

(b) For a convex compact set $\emptyset \neq K \subset \mathbb{R}^{n}$ we define its support function

$$
h_{K}: \mathbb{R}^{n} \longrightarrow \mathbb{R}, h_{K}(\xi):=\sup \{\langle\xi, x\rangle: x \in K\} .
$$

Now we are ready to introduce the Phragmén-Lindelöf conditions, which are studied in the sequel.

Definition 2.5. Let $V \subset \mathbb{C}^{n}$ be an algebraic variety, let $\Omega$ be a convex open set in $\mathbb{R}^{n}$ and let $\omega$ be a weight function. Then $V$ satisfies the condition $\operatorname{PL}(\Omega, \omega)$ if the following holds: For each $K \in \mathcal{K}(\Omega)$ there exists $K^{\prime} \in \mathcal{K}(\Omega)$ such that for each $K^{\prime \prime} \in \mathcal{K}(\Omega)$ there exists $B>0$ such that each $u \in \operatorname{PSH}(V)$ satisfying (1) and (2) also satisfies (3), where: 
(1) $u(z) \leq h_{K}(\operatorname{Im} z)+O(\omega(z)), \quad z \in V$,

(2) $u(z) \leq h_{K^{\prime \prime}}(\operatorname{Im} z), \quad z \in V$,

(3) $u(z) \leq h_{K^{\prime}}(\operatorname{Im} z)+B \omega(z), \quad z \in V$.

In the global case $\Omega=\mathbb{R}^{n}$, condition $\operatorname{PL}\left(\mathbb{R}^{n}, \omega\right)$ is easily seen to be equivalent to:

There exists $A>0$ such that for each $\rho>1$ there exists $B>0$ such that each $u \in \operatorname{PSH}(V)$ satisfying $(1 \mathrm{~g})$ and $(2 \mathrm{~g})$ also satisfies $(3 \mathrm{~g})$, where:

(1g) $u(z) \leq|\operatorname{Im} z|+O(\omega(z)), \quad z \in V$,

$(2 \mathrm{~g}) u(z) \leq \rho|\operatorname{Im} z|, \quad z \in V$,

$(3 \mathrm{~g}) u(z) \leq A|\operatorname{Im} z|+B \omega(z), \quad z \in V$.

Remark. In [21], [23] and in Palamodov [30] it was shown that conditions of the form $\operatorname{PL}(\Omega, \omega)$ characterize when a linear partial differential operator $P(D)$ with constant coefficients admits a continuous linear right inverse on $C^{\infty}(\Omega)$ or $\mathcal{E}_{(\omega)}(\Omega)$ or when a complex of differential operators on $C^{\infty}(\Omega)^{k}$ splits. In these characterizations the functions $u$ which arise naturally are of the special form $u=\log |f|, f$ an entire function on $\mathbb{C}^{n}$. In [22] we have proved that these natural conditions are equivalent to $\operatorname{PL}(\Omega, \omega)$ and that it does not matter whether condition 2.5(1) is required to hold on $V$ or on $\mathbb{C}^{n}$.

The following proposition shows that the component structure of the algebraic variety $V$ is not important, as Hörmander [15] has pointed out already.

Proposition 2.6. An algebraic variety $V$ satisfies $\operatorname{PL}(\Omega, \omega)$ if and only if each irreducible component of $V$ satisfies $\operatorname{PL}(\Omega, \omega)$.

Proof. Let $V_{1}, \ldots, V_{k}$ be the irreducible components of $V$. If $\operatorname{PL}(\Omega, \omega)$ holds for $V_{j}, 1 \leq j \leq k$, then it holds for $V$. Conversely, if $V$ satisfies $\operatorname{PL}(\Omega, \omega)$ and if $V_{m}$ is fixed, then we can find a psh function $v$ on $\mathbb{C}^{n}$ which is $-\infty$ on $V_{j}$ for $j \neq m$, satisfies $\left.v\right|_{V_{m}} \not \equiv-\infty$ and $v(z)=O(\log (1+|z|))$ as $|z| \rightarrow \infty$. Now let $u$ be psh on $V_{m}$ and satisfy 2.5(1) and 2.5(2) on $V_{m}$. Then $u+\varepsilon v$ can be extended to $V$ by defining it as $-\infty$ on $V \backslash V_{m}$. Hence $\operatorname{PL}(\Omega, \omega)$ for $V$ implies that the extended function satisfies 2.5(3) on $V$ and consequently on $V_{m}$. If we let $\varepsilon$ go to zero, we see that $u$ satisfies $2.5(3)$ on $V_{m}$.

Remark 2.7. The condition $\operatorname{PL}(\Omega, \omega)$ is invariant under translation and real linear change $A$ of coordinates, since for every $a, \xi \in \mathbb{R}^{n}: h_{K+a}(\xi)=h_{K}(\xi)+\langle\xi, a\rangle$ and $h_{A(K)}(\xi)=h\left(A^{t} \xi\right)$.

Proposition 2.8. (a) The condition $\mathrm{PL}(\Omega, \omega)$ is not changed if the condition 2.5(1) is replaced by

$(1)^{\prime} u(z) \leq h_{K}(\operatorname{Im} z)+O(1), \quad z \in V$.

(b) The condition $\operatorname{PL}(\Omega, \omega)$ is equivalent to the following one: For each $K \in$ $\mathcal{K}(\Omega)$ there exists $K^{\prime} \in \mathcal{K}(\Omega)$ such that for each $K^{\prime \prime} \in \mathcal{K}(\Omega)$ and each (some) $C \geq 0$ there exists $B>0$ such that each $u \in \operatorname{PSH}(V)$ satisfying 2.5(1) and (2)' also satisfies 2.5(3), where

$(2)^{\prime} u(z) \leq h_{K^{\prime}}(\operatorname{Im} z)+C \omega(z), \quad z \in V$.

Proof. (a) Obviously, it suffices to show that the modified version of $\operatorname{PL}(\Omega, \omega)$ implies the one from 2.5. To do this we use Braun, Meise and Taylor [6], 1.6, to find a weight function $\sigma$ with $\sigma=o(\omega)$. In view of Remark 2.7 we may assume $0 \in \stackrel{\circ}{K} \subset K^{\prime} \subset K^{\prime \prime}$. Hence Lemma 2.2 implies the existence of $\varphi \in \operatorname{PSH}\left(\mathbb{C}^{n}\right)$ 
satisfying $\varphi(z) \leq h_{K}(\operatorname{Im} z)-\sigma(z)$. Now fix $u \in \operatorname{PSH}(V)$ and assume that $u$ satisfies $2.5(1)$ and 2.5(2). Then $\frac{1}{1+\delta}(u+\delta \varphi)$ satisfies (1)' and 2.5(2), and consequently it satisfies 2.5(3). Passing to the limit $\delta=0$, we see that $u$ satisfies $2.5(3)$.

(b) It suffices to show that $\operatorname{PL}(\Omega, \omega)$ implies the present condition (for all $C>0$ ). To do this, fix $K \in \mathcal{K}(\Omega)$ and find $K^{\prime} \in \mathcal{K}(\Omega)$ according to $\operatorname{PL}(\Omega, \omega)$. Again we may assume $0 \in \stackrel{\circ}{K} \subset K^{\prime}$. Now fix $Q \in \mathcal{K}(\Omega)$ with $K^{\prime} \subset \stackrel{\circ}{Q}$. If $K^{\prime \prime} \in \mathcal{K}(\Omega)$ and $C>0$ are given, choose $\varepsilon>0$ so small that $(1+C \varepsilon) K^{\prime} \subset Q$. Next use Lemma 2.2 to find a psh function $\varphi_{\varepsilon}$ on $\mathbb{C}^{n}$ so that

$$
-C_{\varepsilon} \omega(z) \leq \varphi_{\varepsilon}(z) \leq \varepsilon h_{K}(\operatorname{Im} z)-\omega(z) \text { for all } z \in \mathbb{C}^{n} .
$$

Now assume that $u$ is psh on $V$ and satisfies 2.5(1) and $(2)^{\prime}$. Then $\frac{1}{1+C \varepsilon}\left(u+C \varphi_{\varepsilon}\right)$ satisfies 2.5(1) and 2.5(2). Hence it satisfies 2.5(3) by hypothesis, i.e.

$$
\begin{aligned}
u(z) & \leq(1+C \varepsilon)\left[h_{K^{\prime}}(\operatorname{Im} z)+B \omega(z)\right]+C C_{\varepsilon} \omega(z) \\
& \leq h_{Q}(\operatorname{Im} z)+\left[(1+C \varepsilon) B+C C_{\varepsilon}\right] \omega(z) .
\end{aligned}
$$

Corollary 2.9. If an algebraic variety $V$ satisfies $\operatorname{PL}(\Omega, \omega)$, then it satisfies $\operatorname{PL}(\Omega, \sigma)$ for each weight function $\sigma \geq \omega$.

Note that the main ingredient in the Phragmén-Lindelöf condition is that the uniform estimate of the growth in the imaginary directions given in 2.5(2) is improved in 2.5(3). Analogous to a result of Hörmander [15], 2.1, we prove next that an arbitrarily small amount of improvement in the estimate suffices.

Lemma 2.10. Let $V$ be an algebraic variety and let $\Theta>1$. Assume that for some $B>0$, for some compact convex sets $K \subset K^{\prime} \subset \mathbb{R}^{n}$ with $0 \in \stackrel{\circ}{K}$ and for all $u \in \operatorname{PSH}(V)$ we have that (1) and (2l) imply (3), where

(1) $u(z) \leq h_{K}(\operatorname{Im} z)+O(\omega(z)), \quad z \in V$,

(2l) $u(z) \leq \Theta h_{K^{\prime}}(\operatorname{Im} z), \quad z \in V$,

(3) $u(z) \leq h_{K^{\prime}}(\operatorname{Im} z)+B \omega(z), \quad z \in V$.

Then for each $k \in \mathbb{N}, k \geq 2$, and each $\varepsilon>0$ there exists $B(k, \varepsilon)>0$ such that for all $u \in \operatorname{PSH}(V)$ we have that (1) and $(2 \mathrm{l})^{\prime}$ imply $(3)^{\prime}$, where

$(2 \mathrm{l})^{\prime} u(z) \leq \Theta^{k} h_{K^{\prime}}(\operatorname{Im} z), \quad z \in V$,

$(3)^{\prime} u(z) \leq(1+\varepsilon) h_{K^{\prime}}(\operatorname{Im} z)+B(k, \varepsilon) \omega(z), \quad z \in V$.

Proof. The result follows by induction on $k \in \mathbb{N}$. To carry out the induction step, assume that $u \in \operatorname{PSH}(V)$ satisfies (1) and (21)' for $k \in \mathbb{N}, k \geq 2$. Then fix $\varepsilon>0$ and apply the induction hypothesis to $u / \Theta$, to get the existence of $B^{\prime}=B\left(k-1, \frac{\varepsilon}{2}\right)>0$ so that

$$
u(z) \leq \Theta\left(1+\frac{\varepsilon}{2}\right) h_{K^{\prime}}(\operatorname{Im} z)+\Theta B^{\prime} \omega(z) \text { for all } z \in V .
$$

Then choose $\eta>0$ such that

$$
1+\Theta B^{\prime} \eta \leq 1+\frac{\varepsilon}{2}+B^{\prime} \eta \leq 1+\varepsilon
$$

Next use Lemma 2.2 to find $\varphi_{\eta} \in \operatorname{PSH}\left(\mathbb{C}^{n}\right)$ and $C_{\eta}>0$ so that

$$
-C_{\eta} \omega(z) \leq \varphi_{\eta}(z) \leq \eta h_{K}(\operatorname{Im} z)-\omega(z) \text { for all } z \in \mathbb{C}^{n} .
$$


Because of these choices $\left(1+\frac{\varepsilon}{2}+B^{\prime} \eta\right)^{-1}\left(u+\Theta B^{\prime} \varphi_{\eta}\right)$ satisfies (1) and (2l) and hence (3). This implies

$$
\begin{aligned}
u(z) & \leq\left(1+\frac{\varepsilon}{2}+B^{\prime} \eta\right) h_{K^{\prime}}(\operatorname{Im} z)+\left(1+\frac{\varepsilon}{2}+B^{\prime} \eta\right) B \omega(z)-\Theta B^{\prime} \varphi_{\eta}(z) \\
& \leq(1+\varepsilon) h_{K^{\prime}}(\operatorname{Im} z)+\left((1+\varepsilon) B+\Theta B^{\prime} C_{\eta}\right) \omega(z) .
\end{aligned}
$$

Hence $u$ satisfies $(3)^{\prime}$ with $B(k, \varepsilon)=(1+\varepsilon) B+\Theta B^{\prime} C_{\eta}$.

Corollary 2.11. If an algebraic variety $V$ satisfies $\mathrm{PL}(\Omega, \omega)$ for some open convex set $\Omega$ in $\mathbb{R}^{n}$, then it satisfies $\mathrm{PL}\left(\mathbb{R}^{n}, \omega\right)$.

Proof. Without loss of generality we can assume that $\Omega$ contains the closed unit ball $B$. For $K:=B$ choose $K^{\prime}$ according to $\operatorname{PL}(\Omega, \omega)$. Then choose $\Theta>1$ so that $\Theta K^{\prime} \subset \Omega$ and find $A>0$ with $h_{K^{\prime}}(\xi) \leq \frac{A}{2}|\xi|$. Fix $p>1$ and choose $k \in \mathbb{N}$ with $\Theta^{k}>p$. Then it is easy to check that $\operatorname{PL}(\Omega, \omega)$ applied with $K^{\prime \prime}=\Theta K^{\prime}$ together with Lemma 2.10 proves that $(1 \mathrm{~g})$ and $(2 \mathrm{~g})$ imply $(3 \mathrm{~g})$. Hence $\mathrm{PL}\left(\mathbb{R}^{n}, \omega\right)$ holds.

Corollary 2.12. Let $V$ be an algebraic variety in $\mathbb{C}^{n}$ and let $\Omega$ be a bounded open convex set in $\mathbb{R}^{n}$ with $0 \in \Omega$. Then we have:

( $\alpha$ ) If $V$ satisfies $\mathrm{PL}(\Omega, \omega)$, then for every $\varepsilon>0$ and every $\rho>1+\varepsilon$ there exists $B=B(\varepsilon, \rho)$ such that each $u \in \operatorname{PSH}(V)$ which satisfies (1b) and (2b) also satisfies (3b), where

(1b) $u(z) \leq h_{\bar{\Omega}}(\operatorname{Im} z)+O(\omega(z)), \quad z \in V$

(2b) $u(z) \leq \rho h_{\bar{\Omega}}(\operatorname{Im} z), \quad z \in V$,

(3b) $u(z) \leq(1+\varepsilon) h_{\bar{\Omega}}(\operatorname{Im} z)+B \omega(z), \quad z \in V$.

( $\beta$ ) If for every $\varepsilon>0$ there exist $\rho>1+\varepsilon$ and $B=B(\varepsilon, \rho)$ such that each $u \in \operatorname{PSH}(V)$ which satisfies (1b) and (2b) also satisfies (3b), then $V$ satisfies $\operatorname{PL}(\Omega, \omega)$.

Proof. $(\alpha)$ Let $\varepsilon>0$ and $\rho>1+\varepsilon$ be given. Since $\{t \bar{\Omega}\}_{t \in] 0,1[}$ is a fundamental system for the compact subsets of $\Omega$, condition $\operatorname{PL}(\Omega, \omega)$ implies that for each $s \in] 0,1\left[\right.$ there exists $t=t(s)>s$ so that for $K=s \bar{\Omega}$ we can choose $K^{\prime}=t \bar{\Omega}$ according to $\operatorname{PL}(\Omega, \omega)$. Now choose $s$ so large that $\frac{t}{s}<1+\varepsilon$ and choose $\Theta>1$ so that $\Theta t<1$. Then let $K^{\prime \prime}=\Theta t \bar{\Omega}$ and choose $D>0$ according to 2.5. Next fix $u \in \operatorname{PSH}(V)$ satisfying (1b) and

$$
u(z) \leq \Theta \frac{t}{s} h_{\bar{\Omega}}(\operatorname{Im} z) \quad \text { for all } \quad z \in V .
$$

Then su satisfies 2.5(1) and (2) and hence also 2.5(3). This implies

$$
u(z) \leq \frac{t}{s} h_{\bar{\Omega}}(\operatorname{Im} z)+\frac{1}{s} D \omega(z) \text { for all } z \in V .
$$

Now choose $k \in \mathbb{N}$ with $\Theta^{k} \geq \rho$ and choose $\eta>0$ so that $(1+\eta) \frac{t}{s} \leq 1+\varepsilon$. Next fix $u \in \operatorname{PSH}(V)$ satisfying (1b) and (2b). Then Lemma 2.10 implies that for a suitable $B(\varepsilon, \rho)>0$ we have for all $z \in V$

$$
\begin{aligned}
u(z) & \leq(1+\eta) \frac{t}{s} h_{\bar{\Omega}}(\operatorname{Im} z)+B(\varepsilon, \rho) \omega(z) \\
& \leq(1+\varepsilon) h_{\bar{\Omega}}(\operatorname{Im} z)+B(\varepsilon, \rho) \omega(z) .
\end{aligned}
$$

$(\beta)$ If $K \in \mathcal{K}(\Omega)$ is given, find $t \in] 0,1[$ with $t \bar{\Omega} \supset K$. Then choose $\varepsilon>0$ so that $(1+\varepsilon) t<1$ and put $K^{\prime}=(1+\varepsilon) t \bar{\Omega}$. Next fix $K^{\prime \prime} \in \mathcal{K}(\Omega)$ and choose $k \in \mathbb{N}$ so large that $\left(\frac{\rho}{1+\varepsilon}\right)^{k} t \bar{\Omega} \supset K^{\prime \prime}$. Now assume that $u$ satisfies 2.5(1) and (2). Then $u / t$ 
satisfies (1) and $(21)^{\prime}$ of 2.10 if we let $K=K^{\prime}=\bar{\Omega}$ and $\Theta=\frac{\rho}{1+\varepsilon}$. Hence Lemma 2.10 implies

$$
\begin{aligned}
u(z) & \leq(1+\varepsilon) t h_{\bar{\Omega}}(\operatorname{Im} z)+B(k, \varepsilon) \omega(z) \\
& =h_{K^{\prime}}(\operatorname{Im} z)+B(\rho, \varepsilon) \omega(z) z \in V .
\end{aligned}
$$

Remark 2.13. By Proposition 2.8, the condition $\operatorname{PL}(\Omega, \omega)$ is equivalent to the following one: For each $K \in \mathcal{K}(\Omega)$ there exists $K^{\prime} \in \mathcal{K}(\Omega)$ so that for each $\rho>0$ and each $F \geq 0$ there exists $B>0$ such that each $u \in \operatorname{PSH}(V)$ satisfying (1) and (2) also satisfies (3), where

(1) $u(z) \leq h_{K}(\operatorname{Im} z)+O(\omega(z)), \quad z \in V$,

(2) $u(z) \leq \rho|\operatorname{Im} z|+F \omega(z), \quad z \in V$,

(3) $u(z) \leq h_{K^{\prime}}(\operatorname{Im} z)+B \omega(z), \quad z \in V$.

We conclude the present section by the following lemma.

Lemma 2.14. Let $V$ be a pure dimensional algebraic variety of dimension $k$ in $\mathbb{C}^{n}$ and assume that $u \in \mathrm{PSH}(V)$ satisfies

$$
u(z) \leq\|\operatorname{Im} z\|_{1}+O(1) \text { for all } z \in V .
$$

Assume further that for some weight function $\omega$ and some $A, B \geq 1$ the estimate

$$
u(z) \leq A\|\operatorname{Im} z\|_{1}+B \omega(z)
$$

holds for all $z \in V$ for which $k$ of the coordinates of $z=\left(z_{1}, \ldots, z_{n}\right)$ are real. Then for each $\varepsilon>0$ there exists $B_{\varepsilon}>0$ so that

$$
u(z) \leq(A+\varepsilon)\|\operatorname{Im} z\|_{1}+B_{\varepsilon} \omega(z) \text { for all } z \in V .
$$

Proof. Let $P_{\omega}$ denote the harmonic extension of $t \mapsto \omega(|t|)$ to the complex plane. Then [18], 1.5, implies that for each $\varepsilon>0$ there exists $C_{\varepsilon}>0$ so that

$$
\omega(\zeta) \leq P_{\omega}(\zeta) \leq \frac{\varepsilon}{B}|\operatorname{Im} \zeta|+C_{\varepsilon} \omega(\zeta) \text { for all } \zeta \in \mathbb{C} .
$$

If we define $h(z):=\sum_{j=1}^{n} P_{\omega}\left(z_{j}\right)$, then (1) and (3) imply

$$
u(z) \leq A\|\operatorname{Im} z\|_{1}+B h(z)
$$

for all $z \in V$ for which $k$ of the coordinates of $z$ are real. In particular

$$
v(z):=u(z)-A \sum_{j=1}^{n} \operatorname{Im} z_{j}-B h(z)
$$

is psh on $V_{+}:=V \cap\left\{z \in \mathbb{C}^{n}: \operatorname{Im} z_{j}>0\right.$ for $\left.1 \leq j \leq n\right\}$ bounded on $V$ and satisfies $v(z) \leq 0$ for all points in $\partial V_{+}$where $k$ of the coordinates are real. Therefore we have

$$
\sup _{z \in V_{+}} v(z)=\sup _{z \in \partial V_{+}} v(z) .
$$

Replacing $v$ by $v-\eta \sum_{j=1}^{n} \log \left|i+z_{j}\right|$ for $\eta>0$ we can assume that $\sup _{z \in \partial V_{+}} v(z)=$ $v\left(z^{0}\right)$ for some $z^{0}=\left(z_{1}^{0}, \ldots, z_{n}^{0}\right)$ where for $p \leq k$ we have $z_{j}^{0} \in \mathbb{R}$ for $1 \leq j \leq p$. If $p<k$, then

$$
V \cap\left\{z \in \mathbb{C}^{n}: z_{j}=z_{j}^{0} \text { for } 1 \leq j \leq p\right\}
$$

is a variety of dimension at least $k-p \geq 1$ in $\left\{z \in \mathbb{C}^{n}: \operatorname{Im} z_{j}>0\right.$ for $\left.1 \leq j \leq n\right\}$. Hence $v$ must take its maximum at a boundary point. Hence $v\left(z^{0}\right)=v\left(z^{1}\right)$ where $z^{1}=\left(z_{1}^{0}, \ldots, z_{p}^{0}, z_{p+1}^{1}, \ldots, z_{n}^{1}\right)$ and $z_{j}^{1} \in \mathbb{R}$ for some $j$ with $p+1 \leq j \leq n$. Continuing 
in this way we see that there exists $z^{*} \in \partial V_{+}$with $k$ components of $z^{*}$ being real. Hence the hypothesis implies $v\left(z^{*}\right) \leq 0$ and consequently $v(z) \leq 0$ on $V_{+}$. Since the same argument applies to all other cases, we conclude $v(z) \leq 0$ on $V$. Hence (4) holds on $V$ and together with (3) this implies (2).

\section{Homogeneous VARIETIES}

In this section we study the conditions $\operatorname{PL}(\Omega, \omega)$ on homogeneous varieties, i.e. on algebraic varieties $V$ in $\mathbb{C}^{n}$ which satisfy $z \in V$ if and only if $\lambda z \in V$ for all $\lambda \in \mathbb{C}$. For such varieties $V$ we characterize $\operatorname{PL}(\Omega, \omega)$ by various other conditions, including a local Phragmén-Lindelöf condition introduced by Hörmander [15]. As a consequence we get that on homogeneous varieties $\operatorname{PL}(\Omega, \omega)$ is independent of $\omega$ and that we can compare $\operatorname{PL}(\Omega, \omega)$ with the Phragmén-Lindelöf condition $\operatorname{HPL}(\Omega)$ of Hörmander [15]. To formulate one of the main results of this section, we introduce the following definitions.

Definition 3.1. Let $V$ be a homogeneous variety in $\mathbb{C}^{n}$ and $\Omega$ a convex open set in $\mathbb{R}^{n}$. We say $V$ satisfies the condition $\operatorname{PL}(\Omega)$ or $\widetilde{\mathrm{PL}}(\Omega)$ if the following hold:

$\operatorname{PL}(\Omega)$ : For each $K \in \mathcal{K}(\Omega)$ there exists $K^{\prime} \in \mathcal{K}(\Omega)$ such that $u \in \operatorname{PSH}(V)$ satisfying $(1 \mathrm{~h})$ and $(2 \mathrm{~h})$ also satisfies $(3 \mathrm{~h})$, where

(1h) $u(z) \leq h_{K}(\operatorname{Im} z)+o(|z|), \quad z \in V$,

(2h) $u(z) \leq 0, \quad z \in V \cap \mathbb{R}^{n}$,

(3h) $u(z) \leq h_{K^{\prime}}(\operatorname{Im} z), \quad z \in V$.

$\widetilde{\mathrm{PL}}(\Omega)$ : For each $K \in \mathcal{K}(\Omega)$ there exist $K^{\prime} \in \mathcal{K}(\Omega)$ and $\delta>0$ such that each $u \in \operatorname{PSH}(V)$ satisfying (1) and (2) also satisfies (3), where

(1) $u(z) \leq h_{K}(\operatorname{Im} z)+\delta, \quad z \in V$,

(2) $u(z) \leq 0, \quad z \in V \cap \mathbb{R}^{n}$,

(3) $u(z) \leq h_{K^{\prime}}(\operatorname{Im} z), \quad z \in V$.

Also, we introduce the following local Phragmén-Lindelöf condition, which is a variant of the one introduced by Hörmander [15], 4.1.

Definition 3.2. Let $V$ be a homogeneous variety in $\mathbb{C}^{n}$ and $\Omega$ a convex open set in $\mathbb{R}^{n}$. $V$ satisfies $\operatorname{HPL}(\Omega$, loc $)$ at $\xi \in V \cap \mathbb{R}^{n}$, if there are open sets $U_{1} \subset U_{2} \subset U_{3} \subset \subset$ $\mathbb{C}^{n}$ with $\xi \in U_{1}$ such that for each $K \in \mathcal{K}(\Omega)$ there exists $K^{\prime} \in \mathcal{K}(\Omega)$ and $\delta>0$ such that each $u \in \operatorname{PSH}\left(U_{3} \cap V\right)$ satisfying (1) and (2) also satisfies (3), where

(1) $u(z) \leq h_{K}(\operatorname{Im} z)+\delta, \quad z \in U_{3} \cap V$,

(2) $u(z) \leq 0, \quad z \in U_{2} \cap \mathbb{R}^{n} \cap V$,

(3) $u(z) \leq h_{K^{\prime}}(\operatorname{Im} z), \quad z \in U_{1} \cap V$.

Theorem 3.3. Let $V$ be a homogeneous variety in $\mathbb{C}^{n}$ and $\Omega$ a convex open set in $\mathbb{R}^{n}$. Then the following assertions are equivalent:

(1) $V$ satisfies $\operatorname{PL}(\Omega)$.

(2) $V$ satisfies $\operatorname{PL}(\Omega, \omega)$ for each weight function $\omega$ on $\mathbb{C}^{n}$.

(3) V satisfies $\operatorname{PL}(\Omega, \omega)$ for some weight function $\omega$ on $\mathbb{C}^{n}$.

(4) $V$ satisfies $\widetilde{\mathrm{PL}}(\Omega)$.

(5) $V$ satisfies $\operatorname{HPL}(\Omega$, loc $)$ at each $\xi \in V \cap \mathbb{R}^{n}$.

(6) $V$ satisfies $\operatorname{HPL}(\Omega$, loc $)$ at zero.

For the proof of Theorem 3.3 we need the following two lemmas. The first one is taken from $[26], 2.9$. 
Lemma 3.4. There exists $c>0(c=1 / 2$ will do) such that for each $n \in \mathbb{N}$ there exists $H \in C(B) \cap \operatorname{PSH}(\stackrel{\circ}{B}), B:=\left\{x \in \mathbb{C}^{n}:|x| \leq 1\right\}$, having the following properties:

(1) $H(z) \leq|\operatorname{Im} z|$ for $|z| \leq 1$.

(2) $H(z) \leq|\operatorname{Im} z|-c$ for $|z|=1$.

(3) $H(x) \leq 0$ for $x \in \mathbb{R}^{n},|x| \leq 1$.

(4) $H($ iy $) \geq 0$ for $y \in \mathbb{R}^{n},|y| \leq 1$.

Lemma 3.5. Let $V$ be a homogeneous variety in $\mathbb{C}^{n}$, let $\Omega$ be a convex open set in $\mathbb{R}^{n}$ and let $\omega$ be a weight function. If $V$ satisfies $\operatorname{PL}(\Omega, \omega)$, then the following holds:

For each $K \in \mathcal{K}(\Omega)$ there exists $K^{\prime} \in \mathcal{K}(\Omega)$ such that each $u \in \operatorname{PSH}(V)$ satisfying (1) and (2) also satisfies (3), where

(1) $u(z) \leq h_{K}(\operatorname{Im} z)+O(\omega(z)), \quad z \in V$,

(2) $u(z) \leq 0, \quad z \in V \cap \mathbb{R}^{n}$

(3) $u(z) \leq h_{K^{\prime}}(\operatorname{Im} z), \quad z \in V$.

Proof. Let $K \in \mathcal{K}(\Omega)$ be given. Without restriction we can assume $0 \in \Omega$ and $K \supset B=\left\{x \in \mathbb{R}^{n}:|x| \leq 1\right\}$. Choose $Q \supset K$ according to $\operatorname{PL}(\Omega, \omega), \varepsilon>0$ and $\Theta>1$ so that $K^{\prime}:=(1+\varepsilon) Q \in \mathcal{K}(\Omega)$ and $\Theta Q \in \mathcal{K}(\Omega)$. Further, by Braun, Meise and Taylor [6], 1.6, choose a weight function $\sigma$ with $\omega=o(\sigma)$ and use Lemma 2.2 to find $\varphi \in \operatorname{PSH}\left(\mathbb{C}^{n}\right)$ satisfying

$$
\varphi(z) \leq|\operatorname{Im} z|-\sigma(z) \text { for all } z \in \mathbb{C}^{n}
$$

Next assume that $u \in \operatorname{PSH}(V)$ satisfies (1) and (2). Then, for $0<\eta<1$ define

$$
u_{\eta}: V \rightarrow\left[-\infty, \infty\left[, u_{\eta}(z):=(1+\eta)^{-1}(u(z)+\eta \varphi(z))-\eta .\right.\right.
$$

Because $B \subset K$, we get from (4):

For each $0<\eta<1$ there exists $R_{\eta}>0$ so that

In particular,

$$
u_{\eta}(z) \leq h_{K}(\operatorname{Im} z) \text { for all } z \in V \text { with }|z| \geq R_{\eta} .
$$

$$
u_{\eta}(z) \leq h_{K}(\operatorname{Im} z)+O(1), \quad z \in V .
$$

From (2) and (4) we get

$$
u_{\eta}(z) \leq-\eta \quad \text { for all } \quad z \in V \cap \mathbb{R}^{n} \text { with }|z| \leq R_{\eta} .
$$

Since we can assume that $u$ is upper semicontinuous, there is an open set $W_{\eta}$ in $V$ with $W_{\eta} \supset\left\{z \in V \cap \mathbb{R}^{n}:|z| \leq R_{\eta}\right\}$ so that

$$
u_{\eta}(z)<-\eta / 2 \text { for all } z \in V \cap W_{\eta} .
$$

Therefore, we have

$$
\inf \left\{h_{K}(\operatorname{Im} z): z \in V,|z| \leq R_{\eta}, z \notin W_{\eta}\right\}>0 .
$$

Consequently, (5) - (8) imply the existence of $k=k(\eta) \in \mathbb{N}$ with

$$
u_{\eta}(z) \leq \Theta^{k} h_{K}(\operatorname{Im} z) \leq \Theta^{k} h_{Q}(\operatorname{Im} z) \text { for all } z \in V .
$$

Since $u_{\eta}$ satisfies $(6)$ and (9) and since $V$ satisfies $\operatorname{PL}(\Omega, \omega)$, Lemma 2.10 implies the existence of $B=B(\eta, \varepsilon)$ so that

$$
u_{\eta}(z) \leq(1+\varepsilon) h_{Q}(\operatorname{Im} z)+B \omega(z) \quad \text { for all } z \in V .
$$


Next fix $0<r<1$ and define $u_{r, \eta}: z \mapsto \frac{1}{r} u_{\eta}(r z)$. Since $V$ is homogeneous, $u_{r, \eta}$ satisfies (6) and (9). Hence, by the previous argument it satisfies (10), which implies

$$
u_{\eta}(\zeta) \leq(1+\varepsilon) h_{Q}(\operatorname{Im} \zeta)+r B \omega(\zeta / r) \quad \text { for all } \quad \zeta \in V .
$$

Because $\lim _{t \rightarrow \infty} \omega(t) / t=0$, this gives

$$
u_{\eta}(z) \leq(1+\varepsilon) h_{Q}(\operatorname{Im} z) \text { for all } z \in V,
$$

and hence for all $z \in V$,

$$
u(z) \leq(1+\eta)(1+\varepsilon) h_{Q}(\operatorname{Im} z)-\eta \varphi(z)+\eta .
$$

Since $\eta>0$ can be chosen arbitrarily, this implies

$$
u(z) \leq(1+\varepsilon) h_{Q}(\operatorname{Im} z)=h_{K^{\prime}}(\operatorname{Im} z) \text { for all } z \in V .
$$

Proof of Theorem 3.3. (1) $\Longrightarrow(2) \Longrightarrow(3)$ : This is obvious or easy to check.

$(3) \Longrightarrow(4)$ : Fix $K \in \mathcal{K}(\Omega)$ and choose $K^{\prime} \in \mathcal{K}(\Omega)$ according to $\operatorname{PL}(\Omega, \omega)$. Fix $K^{\prime \prime} \in \mathcal{K}(\Omega)$ to get $B>0$ according to $\operatorname{PL}(\Omega, \omega)$. Let $u \in \operatorname{PSH}(V)$ satisfy (1) and (2) of $\widetilde{\mathrm{PL}}(\Omega)$ and note that $u_{r}(z):=\frac{1}{r} u(z r)$ satisfies 3.5(1) and 3.5(2) for each $r>0$. Since $V$ satisfies $\operatorname{PL}(\Omega, \omega)$ by hypothesis, $u_{r}$ satisfies $3.5(3)$. Multiplying through by $r$, using the homogeneity of $h_{K^{\prime}}$ and replacing $r z$ by $z$, this gives

$$
u(z) \leq h_{K^{\prime}}(\operatorname{Im} z)+r B \omega\left(\frac{z}{r}\right), \quad z \in V, \quad r>0 .
$$

Since $\omega(z)=o(|z|)$, we get $u(z) \leq h_{K^{\prime}}(\operatorname{Im} z)$ by passing to the limit $r=0$.

$(4) \Longrightarrow(5)$ : If $K \in \mathcal{K}(\Omega)$ is given, we can assume without restriction that $K \supset$ $B=\left\{x \in \mathbb{R}^{n}:|x| \leq 1\right\}$. Choose $K^{\prime} \in \mathcal{K}(\Omega)$ and $\delta>0$ according to $\widetilde{\mathrm{PL}}(\Omega)$ and find $\lambda>0$ so small that $Q:=(1+\lambda / c) K \in \mathcal{K}(\Omega)$, where $c$ is the constant from Lemma 3.4. Then fix $\varepsilon>0$ and $\xi \in V \cap \mathbb{R}^{n}$. Making $\lambda$ smaller, we can assume that $\delta^{\prime}:=\frac{\lambda \varepsilon}{3} \leq \delta$. Now let $U_{3}:=U_{2}:=U_{\varepsilon}(\xi)$ and $U_{1}:=U_{\varepsilon / 2}(\xi)$ and fix $u \in \operatorname{PSH}\left(U_{\varepsilon}(\xi) \cap V\right)$ which satisfies (1) and (2) of $\operatorname{HPL}(\Omega$, loc $)$ with $\delta$ replaced by $\delta^{\prime}$. To show that $u$ satisfies $(3)$ of $\operatorname{HPL}(\Omega$, loc $)$ with $K$ replaced by $Q$, fix $z_{o} \in U_{\varepsilon / 2}(\xi) \cap V$ arbitrarily. Then $U_{\varepsilon / 2}\left(\operatorname{Re} z_{o}\right) \subset U_{\varepsilon}(\xi)$ and with $H$ denoting the function of Lemma 3.4, we can define $\psi: U_{\varepsilon / 2}\left(\operatorname{Re} z_{o}\right) \cap V \longrightarrow[-\infty, \infty[$ by

$$
\psi(z):=u(z)+\frac{\lambda \varepsilon}{2 c} H\left(\frac{z-\operatorname{Re} z_{o}}{\varepsilon / 2}\right) .
$$

Now Lemma 3.4(2), $K \supset B$ and condition (1) of $\operatorname{HPL}(\Omega$, loc) imply that for each $z \in V$ with $\left|z-\operatorname{Re} z_{o}\right|=\varepsilon / 2$ we have

$$
\psi(z) \leq h_{K}(\operatorname{Im} z)+\delta^{\prime}+\frac{\lambda}{c}|\operatorname{Im} z|-\frac{\lambda \varepsilon}{2}<\left(1+\frac{\lambda}{c}\right) h_{K}(\operatorname{Im} z) .
$$

Hence, the function $\nu: V \longrightarrow[-\infty, \infty[$, defined by

$$
\nu(z):= \begin{cases}\max \left(\psi(z),\left(1+\frac{\lambda}{c}\right) h_{K}(\operatorname{Im} z)\right) & \text { for } z \in V,\left|z-\operatorname{Re} z_{o}\right|<\varepsilon / 2, \\ \left(1+\frac{\lambda}{c}\right) h_{K}(\operatorname{Im} z) & \text { for } z \in V,\left|z-\operatorname{Re} z_{o}\right| \geq \varepsilon / 2,\end{cases}
$$

is psh on $V$. From Lemma 3.4(1) we conclude that

$$
\psi(z) \leq h_{K}(\operatorname{Im} z)+\delta^{\prime}+\frac{\lambda}{c}|\operatorname{Im} z| \leq\left(1+\frac{\lambda}{c}\right) h_{K}(\operatorname{Im} z)+\delta^{\prime}
$$


for all $z \in V$ with $\left|z-\operatorname{Re} z_{o}\right| \leq \varepsilon / 2$. Since $H$ satisfies 3.4(3) and since $u$ satisfies condition (2) of $\operatorname{HPL}(\Omega$, loc $)$, we now see that $\left(1+\frac{\lambda}{c}\right)^{-1} \nu$ satisfies the conditions (1) and $(2)$ of $\widetilde{\mathrm{PL}}(\Omega)$. Hence we obtain

$$
\nu(z) \leq\left(1+\frac{\lambda}{c}\right) h_{K^{\prime}}(\operatorname{Im} z)=h_{Q}(\operatorname{Im} z) \quad \text { for all } z \in V .
$$

From this, $\left|\operatorname{Im} z_{o}\right|<\varepsilon / 2$ and Lemma 3.4(4) we get

$$
h_{Q}\left(\operatorname{Im} z_{o}\right) \geq \nu\left(z_{o}\right) \geq \psi\left(z_{o}\right)=u\left(z_{o}\right)+\frac{\lambda \varepsilon}{2 c} H\left(i \frac{\operatorname{Im} z_{o}}{\varepsilon / 2}\right) \geq u\left(z_{o}\right) .
$$

$(5) \Longrightarrow(6)$ : This is obvious.

$(6) \Longrightarrow(1)$ : Choose zero neighborhoods $U_{1} \subset U_{2} \subset U_{3} \subset \subset \mathbb{C}^{n}$ according to $\operatorname{HPL}(\Omega$, loc $)$ at 0 , fix $K \in \mathcal{K}(\Omega)$, and find $K^{\prime} \in \mathcal{K}(\Omega)$ and $\delta>0$ according to $\operatorname{HPL}(\Omega$, loc $)$. Next let $u \in \operatorname{PSH}(V)$ satisfy $(1 \mathrm{~h})$ and $(2 \mathrm{~h})$ of $\operatorname{PL}(\Omega)$. Then there exists a decreasing function $\varepsilon:\left[0, \infty\left[\longrightarrow \mathbb{R}\right.\right.$ with $\lim _{t \rightarrow \infty} \varepsilon(t)=0$ so that

$$
u(z) \leq h_{K}(\operatorname{Im} z)+\varepsilon(|z|)(1+|z|) .
$$

For $R>1$, define

$$
u_{R}: U_{1} \cap V \longrightarrow\left[-\infty, \infty\left[, \quad u_{R}(z):=\frac{1}{R} u(R z) .\right.\right.
$$

Then the estimate on $u$ implies

$$
u_{R}(z) \leq h_{K}(\operatorname{Im} z)+\frac{1}{R} \varepsilon(R|z|)(1+R|z|) .
$$

To estimate this further, let $D:=\varepsilon(0)$ and assume $R \geq \frac{2 D}{\delta}$. For each $z \in \mathbb{C}^{n}$ with $|z| \leq \frac{\delta}{2 D}$ we then have

$$
\frac{1}{R} \varepsilon(R|z|)(1+R|z|) \leq \frac{D}{R}\left(1+\frac{\delta}{2 D} R\right) \leq \delta .
$$

Next let $\rho:=\sup _{z \in U_{2}}|z|$ and note that for all $z \in U_{2}$ with $|z| \geq \frac{\delta}{2 D}$ we have

$$
\frac{1}{R} \varepsilon(R|z|)(1+R|z|) \leq \frac{1}{R} \varepsilon\left(R \frac{\delta}{2 D}\right)(1+\rho R) \leq \varepsilon\left(R \frac{\delta}{2 D}\right)(1+\rho) .
$$

Since $\lim _{t \rightarrow \infty} \varepsilon(t)=0$, this implies the existence of $R_{o}>1$ so that

$$
u_{R}(z) \leq h_{K}(\operatorname{Im} z)+\delta \text { for all } z \in U_{2} \cap V, \text { all } R \geq R_{o} .
$$

Consequently, $u_{R}$ satisfies the conditions (1) and (2) of $\operatorname{HPL}\left(\Omega\right.$, loc) for all $R \geq R_{o}$. Therefore we get

$$
u_{R}(z) \leq h_{K^{\prime}}(\operatorname{Im} z) \text { for all } z \in U_{1} \cap V \text {, all } R \geq R_{o} .
$$

Now fix $z \in V$ and choose $R \geq R_{o}$ so that $\frac{z}{R}$ is in $U_{1}$. Then $(*)$ gives $u_{R}\left(\frac{z}{R}\right) \leq$ $h_{K^{\prime}}\left(\operatorname{Im} \frac{z}{R}\right)$ and hence $u(z) \leq h_{K^{\prime}}(\operatorname{Im} z)$. Consequently, $u$ satisfies $(3 \mathrm{~h})$ of $\operatorname{PL}(\Omega)$.

Remark 3.6. An examination of the proof of Theorem 3.3 shows that there are several local Phragmén-Lindelöf conditions which can be used in its formulation. E.g. the set $U_{3}$ in the definition of $\operatorname{HPL}(\Omega$, loc $)$ was not used. However, for a characterization of $\operatorname{HPL}(\Omega$, loc $)$ in terms of extremal functions, the present formulation is more convenient. The proof of Theorem 3.3 also shows that one can specify the size of the sets $U_{1}$ and $U_{2}$ and that $U_{1}$ and $U_{2}$ may even depend on $K^{\prime}$ and $\delta$. 
Corollary 3.7. Let $\Omega$ be a bounded, convex open set in $\mathbb{R}^{n}$ and let $V$ be a homogeneous variety in $\mathbb{C}^{n}$. Then $V$ satisfies $\mathrm{PL}(\Omega)$ if and only if each $u \in \operatorname{PSH}(V)$ satisfying $(\alpha)$ and $(\beta)$ also satisfies $(\gamma)$, where

( $\alpha) u(z) \leq h_{\bar{\Omega}}(\operatorname{Im} z)+o(|z|), \quad z \in V$

(३) $u(z) \leq 0, \quad z \in V \cap \mathbb{R}^{n}$,

$(\gamma) u(z) \leq h_{\bar{\Omega}}(\operatorname{Im} z), \quad z \in V$.

Proof. Without loss of generality we can assume $0 \in \Omega$. Then the sets $(t \bar{\Omega})_{0<t<1}$ form a fundamental system in $\mathcal{K}(\Omega)$. Therefore, the condition above implies $\operatorname{PL}(\Omega)$. To prove the converse, fix $s \in] 0,1[$ and choose $t=t(s) \in] 0,1[$ according to $\operatorname{PL}(\Omega)$. Next assume that $u \in \operatorname{PSH}(V)$ satisfies $(\alpha)$ and $(\beta)$. Then $\operatorname{PL}(\Omega)$ implies that $s u$ satisfies $3.1(3 \mathrm{~h})$ with $K^{\prime}=t \bar{\Omega}$, and hence

$$
u(z) \leq h_{\frac{t}{s} \bar{\Omega}}(\operatorname{Im} z) \quad \text { for all } z \in V .
$$

Since $\lim _{s \nearrow 1} \frac{t(s)}{s}=1$, we conclude that $u$ satisfies $(\gamma)$.

Next we want to compare $\mathrm{PL}(\Omega)$ with the Phragmén-Lindelöf condition that was used by Hörmander [15] to characterize the linear partial differential operators $P(D)$ that act surjectively on the space of all real-analytic functions on $\Omega$. Therefore we recall:

Definition 3.8. Let $V$ be a homogeneous variety in $\mathbb{C}^{n}$ and $\Omega$ a convex open set in $\mathbb{R}^{n} . V$ satisfies $\operatorname{HPL}(\Omega)$ if for each $K \in \mathcal{K}(\Omega)$ there exist $K^{\prime} \in \mathcal{K}(\Omega)$ and $\delta>0$ such that each $u \in \operatorname{PSH}(V)$ satisfying $(\alpha)$ and $(\beta)$ also satisfies $(\gamma)$, where

( $\alpha) u(z) \leq h_{K}(\operatorname{Im} z)+\delta|z|, \quad z \in V$,

(ß) $u(z) \leq 0, \quad z \in V \cap \mathbb{R}^{n}$,

$(\gamma) u(z) \leq h_{K^{\prime}}(\operatorname{Im} z), \quad z \in V$.

Proposition 3.9. If a homogeneous variety $V$ in $\mathbb{C}^{n}$ satisfies $\mathrm{PL}(\Omega)$, then it also satisfies $\operatorname{HPL}(\Omega)$.

Proof. Fix $K \in \mathcal{K}(\Omega)$ and assume without loss of generality that $K \supset B:=\{x \in$ $\left.\mathbb{R}^{n}:|x| \leq 1\right\}$. Then choose $K^{\prime} \in \mathcal{K}(\Omega)$ according to $\operatorname{PL}(\Omega)$, let $c$ be the constant from Lemma 3.4 and choose $\delta>0$ so small that $\left(1+\frac{2 \delta}{c}\right) K^{\prime} \subset \Omega$. Next assume that $u \in \operatorname{PSH}(V)$ satisfies the conditions $(\alpha)$ and $(\beta)$ of $\operatorname{HPL}(\Omega)$. To show that $u$ also satisfies $(\gamma)$ of $\operatorname{HPL}(\Omega)$ with $K^{\prime}$ replaced by $\left(1+\frac{2 \delta}{c}\right) K^{\prime}$, fix $z_{o} \in V$, choose $R>2\left|z_{o}\right|$, let $H$ be as in Lemma 3.4 and define

$$
\psi(z):=u(z)+\frac{2 \delta R}{c} H\left(\frac{z-\operatorname{Re} z_{o}}{R}\right), z \in V,\left|z-\operatorname{Re} z_{o}\right| \leq R .
$$

Then we get as in the proof of Theorem $3.3,(4) \Longrightarrow(5)$, that

$$
\psi(z)<\left(1+\frac{2 \delta}{c}\right) h_{K}(\operatorname{Im} z) \quad \text { for all } z \in V,\left|z-\operatorname{Re} z_{o}\right|=R .
$$

Arguing in the same way as in that proof, we obtain

$$
u\left(z_{o}\right) \leq\left(1+\frac{2 \delta}{c}\right) h_{K^{\prime}}\left(\operatorname{Im} z_{o}\right)=h_{\left(1+\frac{2 \delta}{c}\right) K_{K^{\prime}}}\left(\operatorname{Im} z_{o}\right) .
$$

Remark 3.10. If a homogeneous variety $V$ satisfies $\operatorname{HPL}(\Omega)$, then it satisfies $\operatorname{HPL}(\Omega$, loc $)$ at each $\xi \in V \cap \mathbb{R}^{n}, \xi \neq 0$. This can be shown by the same arguments 
which we used in the implication (4) $\Longrightarrow(5)$ of Theorem 3.3, if one chooses $\varepsilon=|\xi| / 2$ and $\delta^{\prime}=\min \left(\frac{\lambda \varepsilon}{3}, \delta \varepsilon\right)$.

In the important case $\Omega=\mathbb{R}^{n}$ the difference between $\operatorname{PL}\left(\mathbb{R}^{n}\right)$ and $\operatorname{HPL}\left(\mathbb{R}^{n}\right)$ can be made completely explicit, using one of the main results of [26]. To do this we recall several dimension conditions from [26], 2.4. For the definition of (real) analytic sets and their dimension we refer to Narasimhan [29].

Definition 3.11. Let $V$ be an algebraic variety in $\mathbb{C}^{n}$ and $\xi \in V \cap \mathbb{R}^{n}$. We say that $V$ satisfies the dimension condition at $\xi$ if for every local irreducible component $W$ of $V$ at $\xi$, the dimension of $W \cap \mathbb{R}^{n}$ as a real analytic variety at $\xi$ is equal to the dimension of $W$ at $\xi$ as a complex variety.

Furthermore we say that $V$ satisfies the strong dimension condition if $V \cap \mathbb{R}^{n} \neq \emptyset$ and, for each $\xi \in V \cap \mathbb{R}^{n}$, the dimension condition holds at $\xi$.

$V$ is said to satisfy the dimension condition if every irreducible component $W$ of $V$ satisfies $\operatorname{dim}_{\mathbb{R}} W \cap \mathbb{R}^{n}=\operatorname{dim}_{\mathbb{C}} W$.

For various characterizations of the dimension condition and the strong dimension condition, we refer to [26]. The following remark is easy to check.

Remark 3.12. A homogeneous variety $V$ in $\mathbb{C}^{n}$ satisfies $\operatorname{HPL}\left(\mathbb{R}^{n}\right.$, loc $)$ at $\xi \in V \cap \mathbb{R}^{n}$ if and only if there exists $A>0$ and open sets $U_{1} \subset U_{2} \subset U_{3} \subset \subset \mathbb{C}^{n}$ such that each $u \in \operatorname{PSH}\left(U_{3} \cap V\right)$ satisfying $0 \leq u \leq 1$ on $U_{3} \cap V$ and $u \leq 0$ on $U_{2} \cap V \cap \mathbb{R}^{n}$ also satisfies $u(z) \leq A|\operatorname{Im} z|$ for all $z \in U_{1} \cap V$.

Theorem 3.13. For a homogeneous variety $V$ in $\mathbb{C}^{n}$ the following assertions are equivalent:

(1) $V$ satisfies $\mathrm{PL}\left(\mathbb{R}^{n}\right)$.

(2) $V$ satisfies $\operatorname{HPL}\left(\mathbb{R}^{n}\right.$, loc $)$ at each $\xi \in V \cap \mathbb{R}^{n}$.

(3) $V$ satisfies the strong dimension condition and $\operatorname{HPL}\left(\mathbb{R}^{n}, \mathrm{loc}\right)$ at each $\xi \in$ $V \cap \mathbb{R}^{n}$

(4) $V$ satisfies the dimension condition and $\operatorname{HPL}\left(\mathbb{R}^{n}\right.$, loc) at each $\xi \in V \cap \mathbb{R}^{n}$ with $|\xi|=1$.

Proof. (1) $\Longrightarrow(2)$ : This follows from Theorem 3.3.

$(2) \Longrightarrow(3)$ : From 3.11 it follows easily that $\operatorname{HPL}\left(\mathbb{R}^{n}\right.$, loc $)$ at $\xi \in V \cap \mathbb{R}^{n}$ implies that the condition $\mathrm{RPL}_{\text {loc }}$ (see [26], 2.3) holds at $\xi$. Hence [26], 2.8, shows that the dimension condition holds at $\xi$.

$(3) \Longrightarrow(4)$ : This holds trivially.

$(4) \Longrightarrow(1)$ : By Proposition 2.6 we may assume that $V$ is irreducible. Then by Theorem 3.3 it suffices to prove the existence of $B>0$ such that each psh function $u$ on $V$ satisfying $u(z) \leq|\operatorname{Im} z|+o(|z|)$ on $V$ and $u \leq 0$ on $V \cap \mathbb{R}^{n}$ also satisfies $u(z) \leq B|\operatorname{Im} z|$ on $V$. To show this, we apply $\operatorname{HPL}\left(\mathbb{R}^{n}\right.$, loc $)$ at each $\xi \in V \cap \mathbb{R}^{n}$ with $|\xi|=1$. Thus we get $A_{\xi}>0$ and open neighborhoods $U_{1}(\xi) \subset U_{2}(\xi) \subset U_{3}(\xi) \subset \subset$ $\mathbb{C}^{n}$ of $\xi$ so that the condition in 3.12 is satisfied. Hence there are $\xi_{1}, \ldots, \xi_{m}$ so that

$$
\left\{x \in V \cap \mathbb{R}^{n}:|x|=1\right\} \quad \subset \bigcup_{j=1}^{m} U_{1}\left(\xi_{j}\right)=: W .
$$

Since $V$ satisfies the dimension condition, we get from [26], 3.2, that the following condition RPL holds: There exists $A \geq 1$ such that each $u \in \operatorname{PSH}(V)$ satisfying $(\alpha)$ and $(\beta)$ also satisfies $(\gamma)$, where 
( $\alpha) u(z) \leq|z|+o(|z|), \quad z \in V$,

(ß) $u(z) \leq 0, \quad z \in V \cap \mathbb{R}^{n}$,

$(\gamma) u(z) \leq A|z|, \quad z \in V$

Fix $u \in \operatorname{PSH}(V)$ which satisfies the conditions stated at the beginning of the proof. Then $u$ satisfies $(\alpha)$ and $(\beta)$. Hence it satisfies $(\gamma)$. Now let $D:=\sup \{|z|$ : $\left.z \in U_{3}\left(\xi_{j}\right), 1 \leq j \leq m\right\}$, fix $1 \leq j \leq m$ and define $v_{R}: U_{3}\left(\xi_{j}\right) \cap V \longrightarrow \mathbb{R}$ by $v_{R}(z)=\max \left(0, \frac{1}{A D R} u(R z)\right)$ for $R>0$. Then $v_{R}$ satisfies $0 \leq v_{R} \leq 1$. Since $\operatorname{HPL}\left(\mathbb{R}^{n} ;\right.$ loc $)$ holds at $\xi_{j}$ we get $v_{R}(z) \leq A^{\prime}|\operatorname{Im} z|$ for all $z \in U_{1}\left(\xi_{j}\right) \cap V$ and consequently, if we let $A^{\prime}:=\max _{1 \leq j \leq m} A_{\xi_{j}}$ :

$$
u(z) \leq A^{\prime} D A|\operatorname{Im} z| \quad \text { for all } z \in R U_{1}\left(\xi_{j}\right) \cap V .
$$

Hence (1) implies that (2) holds for all $z \in\{R W: R>0\} \cap V$. Now note that there exists $M>0$ so that on the complement of this set in $V$, we have $|z| \leq M|\operatorname{Im} z|$. Therefore, we get the desired estimate with $B=\max \left(A M, A^{\prime} D A\right)$.

Remark. Let $P$ be a homogeneous complex polynomial on $\mathbb{C}^{n}$ which is not constant and let $V(P):=\left\{z \in \mathbb{C}^{n}: P(z)=0\right\}$. By Theorem 3.13, $V(P)$ satisfies $\operatorname{PL}\left(\mathbb{R}^{n}\right)$ if and only if $V(P)$ satisfies $\operatorname{dim}_{\mathbb{R}} V(Q) \cap \mathbb{R}^{n}=n-1$ for each irreducible factor $Q$ of $P$ and the condition $\operatorname{HPL}\left(\mathbb{R}^{n}\right.$, loc $)$ at all points $\xi \in V \cap \mathbb{R}^{n},|\xi|=1$. Hence we obtain from Theorem 3.3 and Remark 3.12 together with [21], 4.6, that [21], 4.7, holds.

Corollary 3.14. A homogeneous algebraic variety $V$ in $\mathbb{C}^{n}$ satisfies $\mathrm{PL}\left(\mathbb{R}^{n}\right)$ if and only if $V$ satisfies $\operatorname{HPL}\left(\mathbb{R}^{n}\right)$ and none of its irreducible components $W$ is elliptic (i.e. $\left.W \cap \mathbb{R}^{n}=\{0\}\right)$.

Proof. $\Longrightarrow$ : This is an obvious consequence of Proposition 3.9 and Theorem 3.13.

$\Longleftarrow$ : If $V$ satisfies $\operatorname{HPL}\left(\mathbb{R}^{n}\right)$, then it satisfies $\operatorname{HPL}\left(\mathbb{R}^{n}\right.$, loc $)$ at each $\xi \in V \cap \mathbb{R}^{n}, \xi \neq$ 0 , by Remark 3.10. Now fix an irreducible component $W$ of $V$. Since $W$ is not elliptic by hypothesis, there exists $\xi \in W \cap \mathbb{R}^{n},|\xi|=1$. Since $V$ satisfies $\operatorname{HPL}\left(\mathbb{R}^{n}\right.$, loc $)$ at $\xi$, it also satisfies the condition $\mathrm{RPL}_{\text {loc }}$ at $\xi$ (see [26], 2.3). Therefore, we get from [26], 2.5, that $W$ satisfies $\operatorname{dim}_{\mathbb{R}} W \cap \mathbb{R}^{n}=\operatorname{dim}_{\mathbb{C}} W$. Hence $V$ satisfies the dimension condition. From the above and Theorem 3.13 it now follows that $V$ satisfies $\mathrm{PL}\left(\mathbb{R}^{n}\right)$.

Remark 3.15. For the more refined condition $\operatorname{PL}(\Omega), \Omega$ an open convex set in $\mathbb{R}^{n}$, $\Omega \neq \mathbb{R}^{n}$, the situation is more complicated. There are homogeneous varieties $V$ that satisfy the dimension condition and $\operatorname{HPL}(\Omega)$ but do not satisfy $\operatorname{PL}(\Omega)$. An example is given by the variety

$$
V:=\left\{\left(z_{1}, \ldots, z_{4}\right) \in \mathbb{C}^{4}: z_{1}^{2}+z_{2}^{2}-z_{3}^{2}-z_{4}^{2}=0\right\} .
$$

By [21], Thm.3.8, $V$ fails $\operatorname{PL}(\Omega)$ for each bounded open convex set $\Omega$ in $\mathbb{R}^{n}$ with $C^{1}$-boundary. On the other hand, $\operatorname{HPL}(\Omega)$ holds for these sets $\Omega$ by the converse of Theorem 6.7 of Hörmander [15], p.180, or by Braun [4], Satz 5.4.8.

To derive a further useful corollary from Theorem 3.13 we recall the classical local Phragmén-Lindelöf result which is a consequence of the estimates for the harmonic measure of the half disk, given in [1], Thm. 3.4. 
Proposition 3.16. Let $D_{+}^{n}:=\left\{z=\left(z_{1}, \ldots, z_{n}\right) \in \mathbb{C}^{n}: \operatorname{Im} z_{j} \geq 0,\left|z_{j}\right| \leq 1,1 \leq\right.$ $j \leq n\}$. Then there is a constant $C \geq 1$ such that if $u$ is psh in a neighborhood of $D_{+}^{n}$ and satisfies $(\alpha)$ and $(\beta)$,

$$
\text { ( } \alpha) \quad u(z) \leq 1, \quad z \in D_{+}^{n} ; \quad(\beta) \quad u(z) \leq 0, \quad z \in \mathbb{R}^{n} \cap D_{+}^{n},
$$

then it also satisfies $(\gamma)$, where

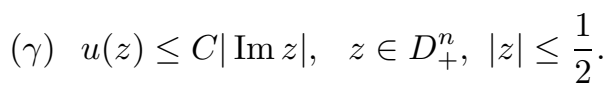

Proof. The function of one complex variable $h(\zeta)=\frac{2}{\pi} \arctan \left(\frac{2 \operatorname{Im} \zeta}{1-|\zeta|^{2}}\right)$ is harmonic in $D_{+}^{1}$ with boundary values 0 on the real axis and 1 on the half-circle. Application of the maximum principle one variable at a time shows that each psh function $u$ on $D_{+}^{n}$ satisfying $(\alpha)$ and $(\beta)$ also satisfies $u(z) \leq h\left(z_{1}\right)+\cdots+h\left(z_{n}\right)$ which clearly implies that $u$ satisfies $(\gamma)$.

Corollary 3.17. Let $V$ be a homogeneous variety in $\mathbb{C}^{n}$. If $V$ satisfies the strong dimension condition and if $\left(V \cap \mathbb{R}^{n}\right) \backslash\{0\} \subset V_{\text {reg }}$, then $V$ satisfies $\mathrm{PL}\left(\mathbb{R}^{n}\right)$.

Proof. By Theorem 3.13 it suffices to show that $V$ satisfies $\operatorname{HPL}\left(\mathbb{R}^{n}\right.$, loc $)$ at each $\xi \in V \cap \mathbb{R}^{n},|\xi|=1$. Since each such $\xi$ is a regular point of $V$ by hypothesis, this can be done as in the proof of [21], 4.8, using Proposition 3.16 and good coordinates for $V$ (see 4.4 below).

Remark 3.18. Let $V$ be a homogeneous variety in $\mathbb{C}^{n}$. In view of Proposition 3.16 and its application in the proof of Corollary 3.17, the only points $\xi \in V \cap \mathbb{R}^{n},|\xi|=$ 1 , at which $\operatorname{HPL}\left(\mathbb{R}^{n}\right.$, loc $)$ might fail are the singular ones. We consider it as an interesting and important problem to understand what happens at these points. The only known results in this direction are due to Hörmander [15] and Braun [3]. By [15], Thm. 6.5, a homogeneous $P \in \mathbb{C}\left[z_{1}, z_{2}, z_{3}\right]$ satisfies $\operatorname{HPL}\left(\mathbb{R}^{3}\right.$, loc $)$ at each $\xi \in V(P) \cap \mathbb{R}^{n},|\xi|=1$, if and only if $P$ is locally hyperbolic at all these points. Braun [3], Cor. 12, shows that this is the case if and only if at $\xi \in V(P) \cap \mathbb{R}^{n},|\xi|=$ $1, V(P)$ satisfies the dimension condition and each irreducible component of the germ $V(P)_{\xi}$ is regular at $\xi$.

\section{Nonhomogeneous VARIETIES, NECESSARY CONDITIONS}

In this section we derive several conditions that are necessary for an algebraic variety to satisfy the Phragmén-Lindelöf condition $\operatorname{PL}(\Omega, \omega)$. First we show that $\operatorname{PL}(\Omega, \omega)$ carries over from an algebraic variety $V$ to its tangent cone $V_{h}$ at infinity. Recall that $V_{h}$ can be defined algebraically as the set of common zeros of the highest order homogeneous terms in the polynomials that vanish on $V$. Or, it may be defined geometrically as the set of all complex lines through the origin that are limits of complex lines through points $z_{j} /\left|z_{j}\right|$, where $z_{j} \in V$ and $\lim _{j \rightarrow \infty}\left|z_{j}\right|=\infty$. (See Chirka [9] for these facts.)

Theorem 4.1. Let $V$ be an algebraic variety in $\mathbb{C}^{n}, \Omega$ a convex open set in $\mathbb{R}^{n}$ and $\omega$ a weight function. If $V$ satisfies $\operatorname{PL}(\Omega, \omega)$, then $V_{h}$ satisfies $\operatorname{PL}(\Omega, \omega)$ and hence $\mathrm{PL}(\Omega)$.

Remark. Let $P$ be a complex polynomial on $\mathbb{C}^{n}$ and denote by $P_{m}$ its principal part. Then the tangent cone at infinity to the variety $V(P):=\left\{z \in \mathbb{C}^{n}: P(z)=0\right\}$ 
is equal to $V\left(P_{m}\right)$. Therefore Theorem 4.1 together with [21], 4.6, shows that the first part of [21], 4.10, holds.

For the proof of Theorem 4.1 we use a method that was developed in [26], sect. 5 , to show that one of the equivalent conditions in Theorem 3.3 holds. To do this we first introduce the appropriate extremal functions and find a suitable estimate for them.

Definition 4.2. Let $D$ be a domain in $\mathbb{C}^{n}, h$ a function on $D, E$ a subset of $D$, and $V$ an analytic variety in $D$. The extremal function of $E$ relative to $h, V$, and $D$ is defined by

$$
\begin{aligned}
U_{E}(z) & :=U_{E}(z ; h, V, D) \\
& :=\sup \{u(z): u \in \operatorname{PSH}(V), u \leq h \text { on } V, u(z) \leq 0, z \in E\} .
\end{aligned}
$$

Lemma 4.3. Let $V$ be an algebraic variety in $\mathbb{C}^{n}, \Omega$ an open convex subset of $\mathbb{R}^{n}$, and $\omega$ a weight function. For $0<\varepsilon<1$ let

$$
K_{\varepsilon}:=\left\{z \in \mathbb{C}^{n}:|z| \leq 2 \text { and }|\operatorname{Im} z| \leq \varepsilon|z| \text { or }|z| \leq \varepsilon\right\}
$$

for $j \in \mathbb{N}$ let

$$
V_{j}:=\{z / j: z \in V\} \text { and let } D:=\left\{z \in \mathbb{C}^{n}:|z|<3\right\} .
$$

If $V$ satisfies the condition $\mathrm{PL}(\Omega, \omega)$, then for each $K \in \mathcal{K}(\Omega)$ there exist $K^{\prime} \in \mathcal{K}(\Omega)$ and $\delta>0$ such that for each $0<\varepsilon<1$ there exists $B_{\varepsilon}>0$ such that for all $j \in \mathbb{N}$ and all $z \in V_{j}$ with $|z|<1$ we have

$$
U_{K_{\varepsilon}}\left(z ; h_{K} \circ \operatorname{Im}+\delta, V_{j}, D\right) \leq h_{K^{\prime}}(\operatorname{Im} z)+j^{-1} B_{\varepsilon} \omega(j z) .
$$

Proof. If $K \in \mathcal{K}(\Omega)$ is given, it is no loss of generality to assume $K \supset\left\{x \in \mathbb{R}^{n}\right.$ : $|x| \leq 1\}$ so that $h_{K} \geq|\cdot|$. Then choose $K_{1} \in \mathcal{K}(\Omega)$ and $0<\eta<1$ so small that

$$
h_{K_{1}}(\operatorname{Im} z) \geq h_{K}(\operatorname{Im} z)+\eta|\operatorname{Im} z| \geq|\operatorname{Im} z| .
$$

Since $V$ satisfies $\operatorname{PL}(\Omega, \omega)$ by hypothesis, we can find $K^{\prime} \in \mathcal{K}(\Omega)$ such that for each $\rho>0$ there exists $B_{\rho}>0$ such that each $\psi \in \operatorname{PSH}(V)$ which satisfies 2.5(1) with $K=K_{1}$ and 2.5(2) with $\rho$ also satisfies

$$
\psi(z) \leq h_{K^{\prime}}(\operatorname{Im} z)+B_{\rho} \omega(z) \text { for all } z \in V .
$$

Next let $c$ denote the constant from Lemma 3.4 and choose $\delta>0$ so small that $\delta<\eta c$. If $0<\varepsilon<1$ is given, choose $\rho_{1}>0$ so large that $h_{K_{1}}(\operatorname{Im} z) \leq \rho_{1}|\operatorname{Im} z|$ and let

$$
\rho:=\rho(\varepsilon):=1+\rho_{1}+\delta \varepsilon^{-2} .
$$

For the proof of the lemma, it clearly suffices to show that each $u \in \operatorname{PSH}\left(V_{j} \cap D\right)$ satisfying

$$
u(z) \leq h_{K}(\operatorname{Im} z)+\delta, z \in V_{j} \cap D \text { and } u(z) \leq 0, z \in K_{\varepsilon} \cap V_{j}
$$

also satisfies

$$
u(z) \leq h_{K^{\prime}}(\operatorname{Im} z)+j^{-1} B_{\rho(\varepsilon)} \omega(j z), z \in V_{j},|z|<1 .
$$

To do this fix $u \in \operatorname{PSH}\left(V_{j} \cap D\right)$ that satisfies (4), and $z_{0} \in V_{j}$ with $\left|z_{0}\right|<1$. Then define $\psi: V \cap\left\{z \in \mathbb{C}^{n}:\left|z-\operatorname{Re}\left(j z_{0}\right)\right|<j\right\} \rightarrow[-\infty, \infty[$ by

$$
\psi(z):=\max \left\{j u\left(\frac{z}{j}\right)+\eta j H\left(\frac{z-\operatorname{Re}\left(j z_{0}\right)}{j}\right), h_{K_{1}}(\operatorname{Im} z)\right\},
$$


where $H$ is the psh function from Lemma 3.4. At points of $V$ on the boundary of this ball, the first term of the maximum does not exeed

$$
\begin{aligned}
j\left(h_{K}\left(j^{-1} \operatorname{Im} z\right)+\delta\right) & +\eta j\left(j^{-1}|\operatorname{Im} z|-c\right) \\
& \leq h_{K}(\operatorname{Im} z)+\eta|\operatorname{Im} z|+(\delta-c \eta) j \\
& \leq h_{K_{1}}(\operatorname{Im} z),
\end{aligned}
$$

by our choice of $K_{1}$. Hence, we can extend $\psi$ to a global psh function on all of $V$ by setting it equal to the second term in the maximum outside this ball. This function obviously satisfies the estimate $2.5(1)$ with $K=K_{1}$. To see that it also satisfies 2.5(2) with $\rho=\rho(\varepsilon)$ we note that outside this ball we have

$$
\psi(z)=h_{K_{1}}(\operatorname{Im} z) \leq \rho_{1}|\operatorname{Im} z| \leq \rho|\operatorname{Im} z| .
$$

For points $z$ inside this ball and $z \in K_{\varepsilon}$ we have $u(z)=0$, so

$$
\begin{aligned}
\psi(z) & \leq \max \left\{\eta j H\left(\frac{z-\operatorname{Re}\left(j z_{0}\right)}{j}\right), h_{K_{1}}(\operatorname{Im} z)\right\} \\
& \leq \max \left\{\eta|\operatorname{Im} z|, h_{K_{1}}(\operatorname{Im} z)\right\} \leq \rho|\operatorname{Im} z|,
\end{aligned}
$$

by 3.4(1). For $z$ inside this ball and $z \notin K_{\varepsilon}$ we have $j \leq|z| / \varepsilon$ and $|z| \leq|\operatorname{Im} z| / \varepsilon$ and consequently

$$
\begin{aligned}
\psi(z) & \leq \max \left\{h_{K}(\operatorname{Im} z)+\frac{\delta}{\varepsilon}|z|, h_{K_{1}}(\operatorname{Im} z)\right\} \\
& \leq h_{K_{1}}(\operatorname{Im} z)+\frac{\delta}{\varepsilon^{2}}|\operatorname{Im} z| \leq \rho|\operatorname{Im} z| .
\end{aligned}
$$

Since $V$ satisfies $\operatorname{PL}(\Omega, \omega)$ the function $\psi$ satisfies $2.5(3)$, i.e.

$$
\psi(z) \leq h_{K^{\prime}}(\operatorname{Im} z)+B_{\rho(\varepsilon)} \omega(z), z \in V .
$$

If we evaluate this inequality at $z=j z_{0} \in V$ and use the fact $H(i y) \geq 0$ for $y \in[-1,1]$, we obtain

$$
j u\left(z_{0}\right) \leq h_{K^{\prime}}(\operatorname{Im} z)+B_{\rho(\varepsilon)} \omega\left(j z_{0}\right) .
$$

Since $z_{0} \in V_{j}$ with $\left|z_{0}\right|<1$ was chosen arbitrarily, this implies (5).

Proof of Theorem 4.1. By Theorem 3.3(6) it suffices to show that $V_{h}$ satisfies the condition $\operatorname{HPL}(\Omega$, loc $)$ at zero. To obtain this as a consequence of the convergence results in [26], we denote by $D$ the ball $\left\{z \in \mathbb{C}^{n}:|z|<3\right\}$ and we let $E:=$ $\mathbb{R}^{n} \cap\left\{z \in \mathbb{C}^{n}:|z| \leq 2\right\}$. Since $V$ satisfies $\operatorname{PL}(\Omega, \omega)$ we get from Lemma 4.3 that for each $K \in \mathcal{K}(\Omega)$ there exist $K^{\prime} \in \mathcal{K}(\Omega)$ and $\delta>0$ such that for each $0<\varepsilon<1$ there exists $B_{\varepsilon}>0$ such that for all $j \in \mathbb{N}$ and all $z \in V_{j}$ with $|z|<1$ we have

$$
U_{K_{\varepsilon}}\left(z ; h_{K} \circ \operatorname{Im}+\delta, V_{j}, D\right) \leq h_{K^{\prime}}(\operatorname{Im} z)+j^{-1} B_{\varepsilon} \omega(j z) \text {. }
$$

If we let $j \rightarrow \infty$ in this estimate and use the fact that the varieties $V_{j}=\{z / j: z \in$ $V\}$ converge to $V_{h}$ in the sense of [26], 4.4, then this theorem and $\lim _{t \rightarrow \infty} \omega(t) / t=0$ imply

$$
U_{K_{\varepsilon}}\left(z ; h_{K} \circ \operatorname{Im}+\delta, V_{h}, D\right) \leq h_{K^{\prime}}(\operatorname{Im} z)
$$

for all regular points $z \in V_{h}$ with $|z|<1$. As $\varepsilon \rightarrow 0$, the compact sets $K_{\varepsilon}$ decrease to the set $E$. Hence it follows from [26], 4.2(1), that for all $z \in\left(V_{h}\right)_{\text {reg }}$ with $|z|<1$ we have

$$
U_{E}\left(z ; h_{K} \circ \operatorname{Im}+\delta, V_{h}, D\right) \leq h_{K^{\prime}}(\operatorname{Im} z)
$$


It is easy to check that this estimate of the extremal function implies the condition $\operatorname{HPL}(\Omega, \operatorname{loc})$ at zero.

Remark. Note that one can also use a modification of the proof of Hörmander [15], Prop. 4.2 , to show that $V_{h}$ satisfies $\operatorname{HPL}(\Omega, \mathrm{loc})$ at zero and hence $\operatorname{PL}(\Omega)$ if $V$ satisfies the condition $\operatorname{PL}(\Omega, \omega)$.

In order to derive a further necessary condition for an algebraic variety to satisfy $\operatorname{PL}\left(\mathbb{R}^{n}, \omega\right)$, we now introduce good coordinates to describe $V$ and $V_{h}$ appropriately.

Good coordinates for $V$ and $V_{h}$ 4.4. Let $V$ be a pure dimensional algebraic variety of dimension $k$ in $\mathbb{C}^{n}$. Then the variety $V_{h}$ also has dimension $k$. Therefore, after a suitable (real linear) change of variables we can choose coordinates $z=(s, w)$ on $\mathbb{C}^{n}, s \in \mathbb{C}^{n-k}, w \in \mathbb{C}^{k}$, so that the projection map $\pi:(s, w) \longmapsto w$ is a proper map of $V$ and $V_{h}$ onto $\mathbb{C}^{k}$. Then we have

$$
\begin{aligned}
& V=\left\{\left(\alpha_{j}(w), w\right): 1 \leq j \leq m\right\}, \\
& V_{h}=\left\{\left(\beta_{j}(w), w\right): 1 \leq j \leq m^{\prime}\right\}
\end{aligned}
$$

where the $\alpha_{j}$ and $\beta_{j}$ are locally multiple-valued analytic functions. Moreover we can assume that the coordinates are such that

$$
\left|\alpha_{j}(w)\right| \leq C(1+|w|) \text { and }\left|\beta_{j}(w)\right| \leq C|w| \text { for all } w \in \mathbb{C}^{k} \text {, all } j
$$

(see for example [9], Thm. 2, p. 77). As $|w| \rightarrow \infty$ the varieties $V$ and $V_{h}$ come close to each other. More precisely, there are constants $\varepsilon>0$ and $C \geq 1$ depending only on $V$ and the choice of coordinates such that for each $w \in \mathbb{C}^{k}$ we have

$$
\begin{aligned}
& \max _{1 \leq j \leq m^{\prime}}\left\{\min _{1 \leq l \leq m}\left|\beta_{j}(w)-\alpha_{l}(w)\right|\right\} \leq C(1+|w|)^{1-\varepsilon}, \\
& \max _{1 \leq l \leq m}\left\{\min _{1 \leq j \leq m^{\prime}}\left|\beta_{j}(w)-\alpha_{l}(w)\right|\right\} \leq C(1+|w|)^{1-\varepsilon} .
\end{aligned}
$$

Also, there is a homogeneous polynomial $D_{h}(w)$ of degree $d$ on $\mathbb{C}^{k}$ such that the branched cover $\pi: V_{h} \rightarrow \mathbb{C}^{k}$ is an analytic cover over $S:=\left\{w \in \mathbb{C}^{k}: D_{h}(w) \neq 0\right\}$ and such that each fiber over $w \in S$ has exactly $m^{\prime}$ distinct points. Since $V_{h}$ is a homogeneous variety, it follows that for each $w \in S$ there exists $\varepsilon_{0}>0$ such that

$$
\left|\beta_{j}(\zeta w)-\beta_{l}(\zeta w)\right| \geq \varepsilon_{0}|\zeta w| \text { for } 1 \leq j, l \leq m^{\prime}, j \neq l, \zeta \in \mathbb{C} .
$$

In particular, for each $\eta>0$ there exists $R_{\eta}>0$ such that for each $w \in \mathbb{C}^{k}$ with $|w|=1$ and $\left|D_{h}(w)\right| \geq \eta$ there exist positive integers $\mu_{j}, 1 \leq j \leq m^{\prime}$, so that for all $\zeta \in \mathbb{C}$ with $|\zeta| \geq R_{\eta}$ and each $1 \leq j \leq m^{\prime}$ there are exactly $\mu_{j}$ of the $\alpha_{l}(w)$ satisfying

$$
\left|\zeta \beta_{j}(w)-\alpha_{l}(\zeta w)\right| \leq C(1+|\zeta w|)^{1-\varepsilon} .
$$

Lemma 4.5. Let $V$ be a pure dimensional algebraic variety in $\mathbb{C}^{n}$ of dimension $k$ and let $V_{h}$ denote its tangent cone at infinity. Then for each irreducible component $W$ of $V_{h}$ there exists a number $\left.a \in\right]-\infty, 1[$ so that the following holds: For each choice of good coordinates for $V$ and $V_{h}$ there are a homogeneous polynomial $\widetilde{D} \not \equiv 0$ on $\mathbb{C}^{k}$ and constants $C, \varepsilon>0$ so that (in the notation of 4.4) for each $\eta>0$ there exist $E, R>0$ such that for each $w \in \mathbb{C}^{k}$ with $|w|=1$ and $|\widetilde{D}(w)| \geq \eta$ and for each $z=\left(\alpha_{l}(\zeta w), \zeta w\right) \in V$ with $|\zeta| \geq R$ there is a unique $j$ with $1 \leq j \leq m^{\prime}$ and

$$
\left|\alpha_{l}(\zeta w)-\zeta \beta_{j}(w)\right| \leq C(1+|\zeta w|)^{1-\varepsilon} .
$$


Moreover, if $\left(\beta_{j}(w), w\right)$ belongs to the component $W$, then $z=\left(\alpha_{l}(\zeta w), \zeta w\right)$ satisfies

$$
\operatorname{dist}\left(z, V_{h}\right) \leq E(1+|z|)^{a} .
$$

If $m=m^{\prime}$, then

$$
a=a(W) \leq 0 \text { for each irreducible component } W \text { of } V_{h} .
$$

Proof. Since we have good coordinates in $\mathbb{C}^{n}$ for $V$ and $V_{h}$, according to 4.4 , we can find a homogeneous polynomial $D_{h}$ and a polynomial $D_{V}$ on $\mathbb{C}^{k}$ so that the projection $\pi:(s, w) \mapsto w$ provides a branched cover of $V_{h}$ and $V$ over $\mathbb{C}^{k}$ which is an analytic cover over $\left\{w \in \mathbb{C}^{k}: D_{h}(w) \neq 0\right\}$ for $V_{h}$ and over $\left\{w \in \mathbb{C}^{k}: D_{V}(w) \neq 0\right\}$ for $V$. Let $Q$ be the highest degree homogeneous term in $D_{V}$ and put $\widetilde{D}:=D_{h} \cdot Q$. Then for each $w \in \mathbb{C}^{k}$ with $\widetilde{D}(w) \neq 0$ and all large $\zeta \in \mathbb{C}$, the functions $\alpha_{l}(\zeta w)$ are locally analytic and single-valued in the $w$-variable. By 4.4 there are $C \geq 1$ and $\varepsilon>0$ so that $4.4(3)$ holds.

Next fix $\eta>0$. Then a compactness argument gives the existence of $R_{0}>1$ and $\delta>0$ so that for each $w_{0} \in \mathbb{C}^{k}$ with $\left|w_{0}\right|=1$ and $\left|\widetilde{D}\left(w_{0}\right)\right| \geq \eta$ we have $\widetilde{D}(\zeta w) \neq 0$ for all $w \in \mathbb{C}^{k}$ with $\left|w-w_{0}\right| \leq \delta$ and all $\zeta \in \mathbb{C}$ with $|\zeta| \geq R_{0}$. By 4.4(4) we can choose $R_{0}$ so large that for each $1 \leq j \leq m^{\prime}$ there are exactly $\mu_{j}$ of the $\alpha_{l}(w)$ satisfying (1) for $|\zeta| \geq R_{0}$. Since $\beta_{j}(w) \neq \beta_{l}(w)$ if $\widetilde{D}(w) \neq 0$ and $j \neq l$, we can choose $\delta>0$ so small that for each $1 \leq j \leq m^{\prime}$ and each $\xi_{0}=\left(\beta_{j}\left(w_{0}\right), w_{0}\right)$ with $\left|\widetilde{D}\left(w_{0}\right)\right| \geq \eta$ and

$$
\begin{gathered}
\Gamma\left(\xi_{0}, \delta, R_{0}\right):=\left\{(s, \zeta w) \in \mathbb{C}^{n}: \zeta \in \mathbb{C},|\zeta|>R_{0},\left|w-w_{0}\right|<\delta,\right. \\
\left.\left|s-\zeta \beta_{j}(w)\right|<\delta|\zeta w|\right\}
\end{gathered}
$$

the set $V \cap \Gamma\left(\xi_{0}, \delta, R_{0}\right)$ has $\mu_{j}$ different branches of $V$ and that the functions $\alpha_{l}(\zeta w)$ that satisfy (1) are analytic and single-valued in $w$ although they may be multiplevalued in $|\zeta|>R_{0}$. Now we fix $j$ and $w$ with $\left|w-w_{0}\right|<\delta$. For each choice of $\alpha_{l}(\zeta w)$ the function $\zeta \mapsto \alpha_{l}(\zeta w)$ can be continued analytically along all paths in $|\zeta|>R_{0}$. Consequently there is an integer $q \leq \mu_{j}$ so that

$$
f_{l}(\tau, w)=\alpha_{l}\left(\tau^{q} w\right), \tau \in \mathbb{C},|\tau|>R_{0}^{1 / q},
$$

is a single-valued analytic function. The Laurent series expansion in $\tau=\zeta^{1 / q}$ is then the Puiseux series expansion of $\alpha_{l}(\zeta w)$. Its coefficients

$$
d_{l, \nu}(w):=\frac{1}{2 \pi i} \int_{|\tau|=\rho} \frac{f_{l}(\tau, w)}{\tau^{\nu+1}} d \tau, \quad \nu \in \mathbb{Z}, \quad \rho=\left(2 R_{0}\right)^{1 / q},
$$

are analytic in $w$ for $\left|w-w_{0}\right|<\delta$. Note that the estimate (1) implies

$$
d_{l, q}(w)=\beta_{j}(w) \text { and } d_{l, \nu}(w)=0 \text { for } \nu>q .
$$

Hence for $\left|w-w_{0}\right|<\delta,|\zeta|>R_{0}$ :

$$
\alpha_{l}(\zeta w)=\zeta \beta_{j}(w)+\sum_{\nu=-\infty}^{q-1} d_{l, \nu}(w) \zeta^{\nu / q} .
$$

Now define

$$
b_{l, j}:=\max \left\{\frac{\nu}{q}: \nu \in \mathbb{Z}, \nu \leq q-1, d_{l, \nu} \not \equiv 0\right\} .
$$

The numbers $b_{l, j}$ depend only on the irreducible component of $V \cap \Gamma\left(\xi_{0}, \delta, R_{0}\right)$ to which $\left(\alpha_{l}(\zeta w), \zeta w\right)$ belongs. There are at most $\mu_{j}$ different values of $b_{l, j}$ corresponding to these different branches. They also seem to depend on $j$. However, 
the numbers are locally constant for $\left(\beta_{j}(w), w\right)$. Therefore they depend only on the connected component to which $\left(\beta_{j}(w), w\right)$ belongs. Hence for the irreducible component $W=W_{i}$ of $V_{h}$, the number $a=a_{i}$ in the assertion is the maximum of the numbers $b_{l, j}$ over all $j$ for which $\left(\beta_{j}(w), w\right)$ is a branch of $W_{i}$ and all $l$ associated with these branches $\left(\beta_{j}(w), w\right)$. If $m=m^{\prime}$ (in the notation of 4.4 ), then $\mu_{j}=1$ for all $j$. Hence the arguments from the beginning of the proof show $q=1$ in (3); i.e. $f_{l}(\tau, w)$ is analytic in $\tau$ and the Puiseux series (4) is its Laurent series for all $l$. In particular, $a_{i} \leq 0$ for each irreducible component $W_{i}$ of $V_{h}$, whenever $m=m^{\prime}$. Hence (3) holds.

Next note that for

$$
D:=\sup \left\{\left|\alpha_{l}\left(\tau^{q} w\right)\right|:|\tau|=\left(2 R_{0}\right)^{1 / q},|w|=1, \text { all } l\right\}
$$

we get from the definition of the Laurent coefficients

$$
\left|d_{l, \nu}(w)\right| \leq D\left(2 R_{0}\right)^{\nu / q} \text { for all } \nu \in \mathbb{Z},|w|=1,\left|w-w_{0}\right|<\delta .
$$

Fix a component $W_{i}$ and choose $j$ and $l$ as before so that $a_{i}=b_{l, j}$. Further, choose $\nu_{0}$ so that $a_{i}=\nu_{0} / q$. From (4) for $w \in \mathbb{C}^{k},|w|=1$ and $\left|w-w_{0}\right|<\delta$ :

$$
\alpha_{l}(\zeta w)=\zeta \beta_{j}(w)+d_{l, \nu_{0}}(w) \zeta^{a_{i}}+\sum_{\nu=-\infty}^{\nu_{0}-1} d_{l, \nu}(w) \zeta^{\nu / q},
$$

where

$$
\left|d_{l, \nu_{0}}(w)\right| \leq D\left(2 R_{0}\right)^{\nu_{0} / q}=E_{1}
$$

and

$$
\left|\sum_{\nu=-\infty}^{\nu_{0}-1} d_{l, \nu}(w) \zeta^{\nu / q}\right| \leq D_{1}|\zeta|^{a_{i}-\frac{1}{q}} \text { for }|\zeta| \geq 3 R_{0} .
$$

In particular, we get for $z=\left(\alpha_{l}\left(\zeta w_{0}\right), \zeta w_{0}\right)$ satisfying (1), where $\left(\beta_{j}\left(w_{0}\right), w_{0}\right)$ belongs to $W_{i}$ and $\left|w_{0}\right|=1,|\zeta| \geq 3 R_{0}$ :

$$
\begin{aligned}
\operatorname{dist}\left(z, V_{h}\right) & \leq\left|\alpha_{l}\left(\zeta w_{0}\right)-\beta_{j}\left(\zeta w_{0}\right)\right| \leq E_{1}|\zeta|^{a_{i}}+D_{1}|\zeta|^{a_{i}-1 / q} \\
& \leq E|\zeta|^{a_{i}} \leq E|z|^{a_{i}}
\end{aligned}
$$

Obviously, this implies (2).

To finish the proof we have to show that $a=a_{i}$ depends only on $W=W_{i}$ and not on the choice of good coordinates. To do this we claim that there exists $\xi_{1} \in W_{\text {reg }}$ with $\left|\xi_{1}\right|=1$ and positive numbers $\sigma_{1}, R_{1}$ and $E$ so that

$$
E^{-1}|z|^{a} \leq \operatorname{dist}\left(z, V_{h}\right) \leq E|z|^{a} \text { for all } z \in V \cap \Gamma,
$$

where $\Gamma=\left\{z \in \mathbb{C}^{n}:|z| \geq R_{1}\right.$ and $\left.\left|\frac{z}{|z|}-\xi_{1}\right| \leq \sigma_{1}\right\}$. To prove our claim fix $w_{0} \in \mathbb{C}^{k}$ and $\xi_{0}=\left(\beta_{j}\left(w_{0}\right), w_{0}\right) \in W$ as before. Assume that $j$ is chosen so that for suitable $l$ we have $a=a(W)=b_{l, j}$. Next choose $w_{1} \in \mathbb{C}^{k}$ satisfying $\left|w_{1}\right| \leq 1$ and $\left|w_{1}-w_{0}\right|<\delta / 2$ such that for some $\varepsilon_{0}>0$ and some $0<\sigma_{2}<\delta / 4$ we have

$$
\left|d_{l, a q}(w)\right| \geq 3 \varepsilon_{0} \text { for all } w \in \mathbb{C}^{k}, \quad|w|=1 \text { and }\left|w-w_{1}\right|<\sigma_{2} .
$$

By (4) and a variant of (6), this implies the existence of $R_{2}>0$ such that

$$
\left|\alpha_{l}(\zeta w)-\beta_{j}(\zeta w)\right| \geq 2 \varepsilon_{0}|\zeta w|^{a} \text { for }|\zeta| \geq R_{2},\left|w-w_{1}\right|<\sigma_{2},|w|=1 .
$$

Now let $\xi_{2}:=\left(\beta_{j}\left(w_{1}\right), w_{1}\right)$ and $\xi_{1}:=\left|\xi_{2}\right|^{-1} \xi_{2}$ and choose $\sigma_{1}>0$ so small and $R_{1}>0$ so large that $\Gamma \subset \Gamma\left(\xi_{2}, \sigma_{2}, R_{2}\right)$. Then the upper estimate in (8) follows from the upper estimate in $(7)$. 
To prove the lower estimate in (8), note that

$$
V_{h} \cap \pi^{-1}\left(\left\{w \in \mathbb{C}^{k}:\left|w-w_{0}\right|<\delta\right\}\right) \subset\left(V_{h}\right)_{\text {reg }} .
$$

Hence there exists $M \geq 1$ such that

$$
\begin{gathered}
\left|\beta_{\nu}(w+h)-\beta_{\nu}(w)\right| \leq M|h| \text { for }\left|w-w_{1}\right| \leq \sigma_{1},|w|=1, \\
|h| \leq \delta / 2,1 \leq \nu \leq m^{\prime} .
\end{gathered}
$$

Now fix $w \in \mathbb{C}^{k},|w|=1,\left|w-w_{1}\right| \leq \sigma_{1}$ and $\Delta \in \mathbb{C}^{k}$. If $|\Delta| \geq \varepsilon_{0} M^{-1}|\zeta w|^{a}$ for $|\zeta| \geq R_{2}$, then

$$
\left|\left(\alpha_{l}(\zeta w), \zeta w\right)-\left(\beta_{j}(\zeta w+\Delta), \zeta w+\Delta\right)\right| \geq|\Delta| \geq \varepsilon_{0}|\zeta w|^{a} .
$$

If $|\Delta| \leq \varepsilon_{0} M^{-1}|\zeta w|^{a}$, then (9), (10) and the homogeneity of $\beta_{j}$ imply

$$
\begin{aligned}
& \left|\left(\alpha_{l}(\zeta w), \zeta w\right)-\left(\beta_{j}(\zeta w+\Delta), \zeta w+\Delta\right)\right| \\
& \quad \geq\left|\alpha_{l}(\zeta w)-\beta_{j}(\zeta w)\right|-\left|\beta_{j}(\zeta w)-\beta_{j}(\zeta w+\Delta)\right| \\
& \quad \geq 2 \varepsilon_{0}|\zeta w|^{a}-M \Delta \geq \varepsilon_{0}|\zeta w|^{a} .
\end{aligned}
$$

From (11) and (12) we get the lower estimate in (8) if we show that the branches $\beta_{\nu}(\zeta w), \nu \neq j$, are further away from $\left(\alpha_{l}(\zeta w), \zeta w\right)$ than $\varepsilon_{0} M|\zeta w|^{a}$. To see this, it suffices to consider points $\left(\beta_{\nu}(\zeta w+\Delta), \zeta w+\Delta\right)$ with $|\Delta| \leq E|\zeta w|^{a}$. Since $\beta_{\nu}(\zeta w)$ and $\beta_{j}(\zeta w)$ for $j \neq \nu$ are apart from each other like $\varepsilon_{1}|\zeta w|$ for some $\varepsilon_{1}>0$ and $\left|w-w_{0}\right|<\delta,|w|=1,|\zeta|>0$, we conclude from (10) and (9) that $\left(\beta_{\nu}(\zeta w+\Delta)\right.$, $\zeta w+\Delta)$ is further away from $\left(\alpha_{l}(\zeta w), \zeta w\right)$ than $\frac{\varepsilon_{1}}{2}|\zeta w|$ for $|\zeta| \geq R_{2}$, provided that $R_{2}$ is large enough. Hence the lower estimate in (8) holds if we choose $E>0$ large enough.

Lemma 4.6. Let $V$ and $V_{h}$ be as in Lemma 4.5, let $W$ be an irreducible component of $V_{h}$ and let a denote the number that is associated to $W$ by Lemma 4.5. Assume that we have chosen good coordinates for $V$ and $V_{h}$ and let $\widetilde{D}$ denote the homogeneous polynomial of Lemma 4.5. If $W$ satisfies the strong dimension condition and if $a>0$, then there exist $\xi_{0}=\left(\beta_{j}\left(w_{0}\right), w_{0}\right) \in W \cap \mathbb{R}^{n}$ with $\left|w_{0}\right|=1$ and $\widetilde{D}\left(w_{0}\right) \neq 0$, and positive numbers $\delta, F$ and $R_{0}$ so that for each $R \geq R_{0}$ we have:

$(\alpha)$ there exists $z(R) \in V$ with $\left|z(R)-R \xi_{0}\right|<F R^{a}$,

( $\beta)|z|^{a}<F|\operatorname{Im} z|$ for all points $z$ that are in the connected component of $V \cap$ $U_{\delta R}\left(R \xi_{0}\right)$ containing $z(R)$.

Proof. Let $\pi: V_{h} \rightarrow \mathbb{C}^{k}$ denote the covering map. Since $W$ satisfies the strong dimension condition and since $\left.\widetilde{D} \circ \pi\right|_{W} \not \equiv 0$ we have that $\left.\widetilde{D} \circ \pi\right|_{W \cap \mathbb{R}^{n}} \not \equiv 0$. Hence we can choose $\xi_{0} \in W \cap \mathbb{R}^{n}$ with $\widetilde{D}\left(\pi\left(\xi_{0}\right)\right) \neq 0$. Then $\xi_{0}=\left(\beta\left(w_{0}\right), w_{0}\right)$ and because of homogeneity, we can assume $\left|w_{0}\right|=1$. Since $\widetilde{D}\left(w_{0}\right) \neq 0$, we get from Lemma 4.5 the existence of $\delta_{0}>0$ and $R_{0}>0$ so that for $w \in \mathbb{C}^{k}$ and $\zeta \in \mathbb{C}$ satisfying $\left|w-w_{0}\right|<\delta_{0}$ and $|\zeta|>R_{0}$ we have the Puiseux expansion for the branches $\alpha_{l}(w)$ of $V$ that are near $\beta$ :

$$
\alpha_{l}(\zeta w)=\zeta \beta(w)+d_{l}(w) \zeta^{a}+g_{l}(\zeta, w)
$$

where $\left|g_{l}(\zeta, w)\right|=O\left(|\zeta|^{b}\right)$ for some $b<a$. Since $d_{l}$ is not identically zero near $w_{0}$, we can assume $d_{l}\left(w_{0}\right) \neq 0$, otherwise we modify $w_{0}$. In fact we can assume $\operatorname{Im} d_{l}\left(w_{0}\right) \neq 0$, otherwise we replace $\zeta$ by $-\zeta$. Now for $R \geq R_{0}$ let

$$
z(R):=\left(\alpha_{l}\left(R w_{0}\right), R w_{0}\right) \in V .
$$


From (1) and 4.5(5), (6) we get the existence of $F>0$ and of $R_{1} \geq R_{0}$ so that

$$
\left|z(R)-R \xi_{0}\right|=\left|\alpha_{l}\left(R w_{0}\right)-\beta\left(R w_{0}\right)\right| \leq\left|d_{l}\left(w_{0}\right) R^{a}+g_{l}\left(R, w_{0}\right)\right|<F R^{a}
$$

for all $R \geq R_{1}$. Hence $(\alpha)$ holds.

To verify $(\beta)$, fix a small number $\delta>0$. Then there exists $\sigma=\sigma(\delta)>0$ so that for sufficiently large $R_{1}$ and each $R \geq R_{1}$ the set

$$
\pi\left(V \cap\left\{z \in \mathbb{C}^{n}:\left|z-R \xi_{0}\right|<\delta R\right\}\right)
$$

is contained in

$$
\left\{r e^{i t} w:|w|=1,\left|w-w_{0}\right| \leq \delta,(1-\delta) R \leq r \leq(1+\delta) R,|t| \leq \sigma(\delta)\right\},
$$

where $\sigma(\delta)$ tends to zero as $\delta$ goes to zero. From this and from $\operatorname{Im} d_{l}\left(w_{0}\right) \neq 0$ we conclude that we can choose $0<\delta<\delta_{0} / 2$ so small that for some constant $B>0$

$$
\left|\operatorname{Im} d_{l}(w) \zeta^{a}\right| \geq \frac{1}{B}|\zeta|^{a} \text { whenever }\left|w-w_{0}\right| \leq \delta,|w|=1,|\arg \zeta| \leq \sigma(\delta) .
$$

Next we note that $W$ is a manifold near $\xi_{0}$ and is the graph of the locally analytic function $\beta$. Since $W$ satisfies the strong dimension condition, $\beta(w)$ is real when $w$ is real and satisfies $\left|w-w_{0}\right|<\delta_{0}$. Hence the ordinary local Phragmén-Lindelöf condition 3.16 implies the existence of a constant $A>0$ so that

$$
|\operatorname{Im} \beta(w)| \leq A|\operatorname{Im} w| \quad \text { for }\left|w-w_{0}\right|<\delta_{0} / 2 .
$$

Finally fix $w \in \mathbb{C}^{k},|w|=1,\left|w-w_{0}\right| \leq \delta$ and $\zeta \in \mathbb{C},|\zeta| \geq R$ and $|\arg \zeta| \leq \sigma(\delta)$ and let $z=\left(\alpha_{l}(\zeta w), \zeta w\right)$. Then we get from (1), (2), (3), and $\left|\alpha_{l}(\zeta w)\right| \leq C|\zeta w|$ for $|\zeta| \geq R$, with $C_{1}:=1+C$

$$
\begin{aligned}
|z|^{a} & \leq C_{1}|\zeta|^{a} \leq B C_{1}\left|\operatorname{Im} d_{l}(w) \zeta^{a}\right| \\
& \leq B C_{1}\left(\left|\operatorname{Im}\left(\zeta \beta(w)+d_{l}(w) \zeta^{a}\right)\right|+|\operatorname{Im}(\zeta \beta(w))|\right) \\
& \leq B C_{1}\left(|\operatorname{Im} z|+O\left(|\zeta|^{b}\right)+A|\operatorname{Im} \zeta w|\right) \\
& \leq B C_{1}(1+A)|\operatorname{Im} z|+O\left(|z|^{b}\right) .
\end{aligned}
$$

Since $b<a$, this implies $(\beta)$, if we choose $F$ large enough.

For the further evaluation of Lemma 4.6, we need the following lemma.

Lemma 4.7. Let $V$ be an algebraic variety in $\mathbb{C}^{n}$ that satisfies $\mathrm{PL}\left(\mathbb{R}^{n}, \omega\right)$ with constants $A>0$ and $B_{\rho}>0$ for $\rho>0$, according to 2.5. Assume that for some $M \geq 1$ and some $z_{0} \in V$ we have $\left|\operatorname{Im} z_{0}\right| \leq M|\operatorname{Im} z|$ for all $z$ in the connected component $\widetilde{V}_{z_{0}}$ of $z_{0}$ in the set $V \cap\left\{z \in \mathbb{C}^{n}:\left|z-z_{0}\right|<t\left|\operatorname{Im} z_{0}\right|\right\}$, where $t \geq \frac{A+2}{c}$ and $c$ is the number in Lemma 3.4. Then $z_{0}$ satisfies

$$
\left|\operatorname{Im} z_{0}\right| \leq B_{(A+2) M+1} \omega\left(z_{0}\right) .
$$

Proof. Let $H$ be the function from Lemma 3.4 and define $u: \widetilde{V}_{z_{0}} \rightarrow \mathbb{R}$ by

$$
u(z):=\max \left\{(A+1)\left|\operatorname{Im} z_{0}\right|+t\left|\operatorname{Im} z_{0}\right| H\left(\frac{z-z_{0}}{t\left|\operatorname{Im} z_{0}\right|}\right),|\operatorname{Im} z|\right\} .
$$

For $z \in V$ with $\left|z-z_{0}\right|=t\left|\operatorname{Im} z_{0}\right|$, by $3.4(2)$, the first term in the maximum is dominated by

$$
(A+1)\left|\operatorname{Im} z_{0}\right|+|\operatorname{Im} z|+\left|\operatorname{Im} z_{0}\right|-t c\left|\operatorname{Im} z_{0}\right| \leq|\operatorname{Im} z| .
$$


Hence we can extend $u$ to a psh function on $V$ by defining it as $u(z)=|\operatorname{Im} z|$ outside $\widetilde{V}_{z_{0}}$. Then $u$ satisfies the condition $2.5(1 \mathrm{~g})$. Moreover, on $\widetilde{V}_{z_{0}}$ we get from 3.4(1) and the hypothesis, that the first term in the maximum is dominated by

$$
(A+1) M|\operatorname{Im} z|+|\operatorname{Im} z|+M|\operatorname{Im} z| \leq((A+2) M+1)|\operatorname{Im} z| .
$$

Hence $u$ also satisfies condition $2.5(2 \mathrm{~g})$ for $\rho=(A+2) M+1$ and consequently $(3 \mathrm{~g}) u(z) \leq A|\operatorname{Im} z|+B_{\rho} \omega(z)$,

since $V$ satisfies $\operatorname{PL}\left(\mathbb{R}^{n}, \omega\right)$. If we apply this at $z=z_{0}$ and note that $H(0)=0$ we get

$$
(A+1)\left|\operatorname{Im} z_{0}\right| \leq u\left(z_{0}\right) \leq A\left|\operatorname{Im} z_{0}\right|+B_{(A+2) M+1} \omega\left(z_{0}\right),
$$

which implies the desired estimate.

Proposition 4.8. Let $V$ be a pure dimensional algebraic variety in $\mathbb{C}^{n}$ of dimension $k$ and let $V_{h}$ denote its tangent cone at infinity. Let $W_{1}, \ldots, W_{p}$ be the irreducible components of $V_{h}$, denote by $a_{i}$ the number that is associated with $W_{i}$ for $1 \leq i \leq p$ according to 4.5 , and let $a:=\max _{1 \leq i \leq p} a_{i}$. If $V$ satisfies the condition $\mathrm{PL}\left(\mathbb{R}^{n}, \omega\right)$ for some weight function $\omega$, then $\omega$ satisfies

$$
R^{a}=O(\omega(R)) \text { for } R \rightarrow \infty .
$$

Proof. If $a \leq 0$, then there is nothing to prove. Hence assume $a>0$ and choose a component $W=W_{i_{0}}$ that satisfies $a=a_{i_{0}}$. Since $V$ satisfies $\operatorname{PL}\left(\mathbb{R}^{n}, \omega\right), V_{h}$ satisfies $\operatorname{PL}\left(\mathbb{R}^{n}\right)$ by Theorem 4.1. Hence $W$ satisfies the strong dimension condition by Theorem 3.13. Introduce good coordinates for $V$ and $V_{h}$ and obtain from Lemma 4.6 the existence of $F>0$, of $\xi_{0}=(\beta(w), w) \in W \cap \mathbb{R}^{n}$ and of points $z(R) \in V, R \geq R_{0}$, that have all the properties stated in 4.6. Next let $A>0$ and $B_{\zeta}$ for $\zeta>0$ denote the constants in $\operatorname{PL}\left(\mathbb{R}^{n}, \omega\right)$ as it holds for $V$ by hypothesis. Let $t:=(A+2) / c$ where $c$ is the constant from Lemma 3.4. We may assume that $R_{0}$ is so large that $(t+1) F R_{0}^{a}<\delta R_{0}$, where $F \geq 1$ and $\delta>0$ are the constants from Lemma 4.6. Note that Lemma $4.6(\alpha)$ and $\xi_{0} \in \mathbb{R}^{n}$ imply

$$
|\operatorname{Im} z(R)|=\left|\operatorname{Im}\left(z(R)-R \xi_{0}\right)\right| \leq F R^{a} \text { for all } R \geq R_{0} .
$$

Now fix $R \geq R_{0}$ and denote by $V(R)$ the connected component of $z(R)$ in the set

$$
V \cap\left\{z \in \mathbb{C}^{n}:|z-z(R)|<t|\operatorname{Im} z(R)|\right\} .
$$

By (1) and 4.6( $\alpha)$ each $z \in V(R)$ satisfies

$$
\begin{aligned}
\left|z-R \xi_{0}\right| & \leq|z-z(R)|+\left|z(R)-R \xi_{0}\right| \\
& \leq t|\operatorname{Im} z(R)|+F R^{a}=(t+1) F R^{a}<\delta R,
\end{aligned}
$$

and hence

$$
|z| \geq\left|R \xi_{0}\right|-\left|z-R \xi_{0}\right| \geq(1-\delta) R
$$

Consequently, 4.6( $\beta)$ holds for all $z \in V(R)$. Applying $4.6(\beta)$ at $z=z(R)$ and using (1), we see

$$
|z(R)|^{a} \leq F|\operatorname{Im} z(R)| \leq F^{2} R^{a} .
$$

Combining (1), (3) and 4.6( $\beta)$, we get for all $z \in V(R)$ :

$$
|\operatorname{Im} z(R)| \leq F R^{a} \leq F(1-\delta)^{-a}|z|^{a} \leq F^{2}(1-\delta)^{a}|\operatorname{Im} z| .
$$

Hence we can apply Lemma 4.7 with $M=F^{2}(1-\delta)^{-a}$ to get $B>0$ such that

$$
|\operatorname{Im} z(R)| \leq F B \omega(z(R)) \text { for all } R \geq R_{0} .
$$


Now choose $B_{1}>0$ so that $\omega\left(F^{2 / a} R\right) \leq B_{1} \omega(R)$ holds for $R \geq R_{0}$. Then (3) applied to $z=z(R)$ together with $4.6(\beta),(5)$ and (3) gives for all $R \geq R_{0}$ :

$$
\begin{aligned}
R^{a} \leq(1-\delta)^{-a}|z(R)|^{a} & \leq(1-\delta)^{-a}|\operatorname{Im} z(R)| \\
& \leq F(1-\delta)^{-a} B \omega\left(F^{2 / a} R\right) \leq F(1-\delta)^{-a} B B_{1} \omega(R) .
\end{aligned}
$$

The following example will show that the necessary condition in Proposition 4.8 is not sufficient.

Example 4.9. For $\lambda \in \mathbb{C}$ with $|\lambda|=1$ we define

$$
P_{\lambda}\left(z_{1}, z_{2}, z_{3}\right):=\lambda z_{1}+z_{2} z_{3}, Q\left(z_{1}, z_{2}, z_{3}\right):=z_{2} z_{3},
$$

and let $V\left(P_{\lambda}\right)=\left\{z \in \mathbb{C}^{3}: P(-z)=0\right\}$. Then the following assertions hold:

(1) $V\left(P_{\lambda}\right)$ satisfies $\mathrm{PL}\left(\mathbb{R}^{3}, \omega\right)$ for each $\lambda \in \mathbb{C}$ with $|\lambda|=1$ and each weight function $\omega$ for which $t^{1 / 2}=O(\omega(t))$ as $t$ tends to infinity.

(2) $V\left(P_{1}\right)$ satisfies $\mathrm{PL}\left(\mathbb{R}^{3}, \log (2+t)\right)$.

(3) $V\left(P_{i}\right)$ fails $\operatorname{PL}\left(\mathbb{R}^{3}, \omega\right)$ for each weight function $\omega$ that satisfies $\omega(t)=o\left(t^{1 / 2}\right)$.

(4) For each $\lambda \in \mathbb{C}$ with $|\lambda|=1$ the number $a(\lambda)$ defined in Proposition 4.8 equals zero.

(5) There exists $M \geq 1$ such that for all $\lambda \in \mathbb{C}$ with $|\lambda|=1$ :

$$
\operatorname{dist}(z, V(Q)) \leq M|z|^{1 / 2} \text { for all } z \in V\left(P_{\lambda}\right) \text { with }|z| \geq 1,
$$

and there is a sequence $\left(z_{j}\right)_{j \in \mathbb{N}}$ in $V\left(P_{\lambda}\right)$ satisfying $\left|z_{j}\right| \rightarrow \infty$ and $M^{-1}\left|z_{j}\right|^{1 / 2} \leq \operatorname{dist}\left(z_{j}, V(Q)\right)$ for all $j \in \mathbb{N}$.

Proof. (1) Since the principal part $Q$ of $P_{\lambda}$ is hyperbolic with respect to $N=$ $(0,1,1)$, we get from [27], 2.12, that $P_{\lambda}$ is $(\omega)$ - hyperbolic with respect to $N$ for each weight function $\omega$ that satisfies $t^{1 / 2}=O(\omega(t))$. Hence $V\left(P_{\lambda}\right)$ satisfies $\operatorname{PL}\left(\mathbb{R}^{3}, \omega\right)$ for all these $\omega$, by [23], 5.4.

(2) Let $u$ be a psh function on $V\left(P_{1}\right)$ that for some $\rho>0$ satisfies

$$
u(z) \leq\|\operatorname{Im} z\|_{1}+O(1) \text { and } u(z) \leq \rho|\operatorname{Im} z| \text { for all } z \in V\left(P_{1}\right) .
$$

To apply Lemma 2.14, we note that for $z \in V\left(P_{1}\right)$ with $z_{2}, z_{3} \in \mathbb{R}$, necessarily $z_{1}$ has to be real. Hence $(*)$ implies $u(z) \leq 0$. If $z$ is in $V\left(P_{1}\right)$ with $z_{1}$ and $z_{2}$ being real, then $z_{3}$ is real except for $z_{2}=0$. In the first case we get $u(z) \leq 0$ as before. In the second case $z=(0,0, \zeta) \in V\left(P_{1}\right)$ for all $\zeta \in \mathbb{C}$. Hence $(*)$ and the regular Phragmén-Lindelöf theorem imply $u(0,0, \zeta) \leq|\operatorname{Im} \zeta|$, which gives $u(z) \leq\|\operatorname{Im} z\|_{1}$ for all $z \in V\left(P_{1}\right)$ with $z_{1}, z_{2} \in \mathbb{R}$. In the same way we get this estimate if $z$ is in $V\left(P_{1}\right)$ and $z_{1}$ and $z_{3}$ are real. Hence Lemma 2.14 implies the existence of some $B>0$ so that

$$
u(z) \leq 2\|\operatorname{Im} z\|_{1}+B \log (2+|z|) \text { for all } z \in V\left(P_{1}\right) .
$$

By Proposition 2.8, this proves that $V\left(P_{1}\right)$ satisfies $\mathrm{PL}\left(\mathbb{R}^{3}, \log (2+t)\right)$.

(3) Assume that $\omega$ is a weight function and that $V\left(P_{i}\right)$ satisfies $\operatorname{PL}\left(\mathbb{R}^{3}, \omega\right)$ with constants $A>0$ and $B_{\rho}>0$ for $\rho>0$. To apply Lemma 4.7, let $t:=(A+2) / c$, where $c$ is the constant from Lemma 3.4 and for $R>0$ we let

$$
z_{R}:=\left(R^{2}, e^{i \frac{\pi}{4}} R, e^{i \frac{\pi}{4}} R\right), V_{R}:=V\left(P_{i}\right) \cap U_{t\left|\operatorname{Im} z_{R}\right|}\left(z_{R}\right) .
$$

Then choose $\varepsilon>0$ so small that

$$
\sin ^{2} \varphi+\cos ^{2}(\varphi-\alpha) \geq \frac{1}{4} \text { for }|\alpha| \leq \varepsilon \text { and } \varphi \in \mathbb{R}
$$


and choose $R_{0}>0$ so large that

$$
\frac{t}{R_{0}} \leq \frac{1}{2} \text { and }|\arg \zeta| \leq \varepsilon \text { for all } \zeta \in \mathbb{C} \text { with }|\zeta-1|<\frac{t}{R_{0}}
$$

Next fix $R \geq R_{0}$ and $z=\left(s, w_{1}, w_{2}\right) \in V_{R}$. Then $\left|\operatorname{Im} z_{R}\right|=R$ implies

$$
\max \left(\left|w_{1}\right|,\left|w_{2}\right|\right) \leq(t+1) R
$$

and hence

$$
\frac{R^{2}}{2} \leq R^{2}\left(1-\frac{t}{R}\right) \leq|s|=\left|w_{1} w_{2}\right| \leq\left|w_{1}\right|(t+1) R
$$

This gives $\left|w_{1}\right| \geq \frac{R}{2(t+1)}$ and similarly $\left|w_{2}\right| \geq \frac{R}{2(t+1)}$. Next note that $z \in V_{R}$ implies $\left|s-R^{2}\right| \leq t\left|\operatorname{Im} z_{R}\right|=t R$ and hence $\left|\frac{s}{R^{2}}-1\right| \leq \frac{t}{R}$. Consequently $s=|s| e^{i \alpha}$ for some $\alpha \in \mathbb{R}$ satisfying $|\alpha| \leq \varepsilon$. If we let $w_{1}=\left|w_{1}\right| e^{i \varphi}, w_{2}=\left|w_{2}\right| e^{i \psi}$, then

$$
|s| \exp (i \alpha)=s=-i w_{1} w_{2}=\left|w_{1} w_{2}\right| \exp (i(\varphi+\psi-\pi / 2))
$$

and consequently

$$
\sin \psi=\sin \left(\alpha+\frac{\pi}{2}-\varphi\right)=\cos (\varphi-\alpha)
$$

By our choice of $\varepsilon$, this implies

$$
\begin{aligned}
|\operatorname{Im} z| \geq\left|\operatorname{Im}\left(w_{1}, w_{2}\right)\right| & \geq \frac{R}{2(t+1)}\left(\sin ^{2} \varphi+\sin ^{2} \psi\right)^{1 / 2} \\
& \geq \frac{R}{4(t+1)}=\frac{1}{4(t+1)}\left|\operatorname{Im} z_{R}\right| .
\end{aligned}
$$

Hence we get from Lemma 4.7

$$
R=\left|\operatorname{Im} z_{R}\right| \leq B_{(A+2) 4(t+1)+1} \omega\left(z_{R}\right) \leq B^{\prime} \omega\left(R^{2}\right) \text { for all } R \geq R_{0} .
$$

This implies $R^{1 / 2}=O(\omega(R))$ for $R \rightarrow \infty$ and proves (3) by contraposition.

(4) We fix $\lambda$ and introduce the coordinates

$$
s:=\left(z_{2}-z_{3}\right) / 2 \quad, \quad w_{1}:=\left(z_{2}+z_{3}\right) / 2 \quad, \quad w_{2}:=z_{1} .
$$

In these coordinates we have

$$
P_{\lambda}\left(s, w_{1}, w_{2}\right)=w_{1}^{2}+\lambda w_{2}-s^{2}, Q\left(s, w_{1}, w_{2}\right)=w_{1}^{2}-s^{2} .
$$

In the sense of 4.4 they are good coordinates for $P_{\lambda}$ and $Q$ and give

$$
\begin{aligned}
& \beta_{1}\left(w_{1}, w_{2}\right)=w_{1}, \quad \beta_{2}\left(w_{1}, w_{2}\right)=-w_{1}, \\
& \alpha_{1}\left(w_{1}, w_{2}\right)=\left(w_{1}^{2}+\lambda w_{1}\right)^{1 / 2}, \alpha_{2}\left(w_{1}, w_{2}\right)=-\left(w_{1}^{2}+\lambda w_{1}\right)^{1 / 2} .
\end{aligned}
$$

This shows $m=m^{\prime}$ in these good coordinates. By Lemma 4.5(3), this implies $a(\lambda) \leq 0$. Computing the Puiseux expansion explicitly, we get $a(\lambda)=0$.

(5) The upper bound for $\operatorname{dist}(z, V(Q))$ in (5) follows easily from Lemma 5.7 below, or by direct computation, using the fact that

$$
V(Q)=\left\{(s, 0, t):(s, t) \in \mathbb{C}^{2}\right\} \cup\left\{(s, t, 0):(s, t) \in \mathbb{C}^{2}\right\} .
$$

To show the estimate from below let $z(\tau)=\left(\bar{\lambda} \tau^{2}, \tau, \tau\right)$ for $\tau \geq 1$. Then $z(\tau)$ is in $V\left(P_{\lambda}\right)$ and $\|z(\tau)\|_{\infty}=\tau^{2}$. For $(s, t) \in \mathbb{C}^{2}$ we have

$$
\|z(\tau)-(s, 0, t)\|_{\infty}=\max \left(\left|\bar{\lambda} \tau^{2}-s\right|,|\tau|,|t-\tau|\right)=\|z(\tau)-(s, t, 0)\|_{\infty} .
$$


It is easy to check that this implies

$$
\inf \left\{\|z(\tau)-z\|_{\infty}: z \in V(Q)\right\} \geq \frac{1}{2}\|z(\tau)\|_{\infty}^{1 / 2},
$$

which implies the second assertion in (5).

Remark 4.10. A straightforward variation of the arguments used in the proofs of $4.9(2)$ and $4.9(3)$ shows that $V\left(P_{-1}\right)$ satisfies $\mathrm{PL}\left(\mathbb{R}^{3}, \log (2+t)\right)$ and that $V\left(P_{\lambda}\right)$ fails $\operatorname{PL}\left(\mathbb{R}^{3}, \omega\right)$ for each weight function $\omega$ which satisfies $\omega(t)=o\left(t^{1 / 2}\right)$ whenever $\operatorname{Im} \lambda \neq 0$.

Remark 4.11. Note that the necessary condition in Proposition 4.8 is not sufficient. To see this, let $P_{i}$ be defined as in 4.9. Then the number $a$, defined in Proposition 4.8 , equals zero by $4.9(4)$. Hence $\omega(t)=\log (2+t)$ satisfies the necessary condition of 4.8 . However, by $4.9(3)$, the variety $V\left(P_{i}\right)$ does not satisfy $\mathrm{PL}\left(\mathbb{R}^{3}, \log (2+t)\right)$.

\section{Nonhomogeneous VARIETIES, SUfFicient CONDITIONS}

In this section we derive a sufficient condition for algebraic varieties to satisfy $\operatorname{PL}\left(\mathbb{R}^{n}, \omega\right)$. This result is used to show that for pure dimensional algebraic varieties $V$ whose tangent cone $V_{h}$ at infinity is a manifold outside the origin, $V$ satisfies $\operatorname{PL}\left(\mathbb{R}^{n}, \omega\right)$ if and only if $V_{h}$ satisfies $\operatorname{PL}\left(\mathbb{R}^{n}\right)$ and if $\operatorname{dist}\left(z, V_{h}\right)=O(\omega(z))$ for $z \in V$ and $|z|$ tending to infinity. As Example 4.9 shows, this does not hold in general if $V_{h}$ has singularities outside the origin.

To formulate our necessary condition, we need the following definition that goes back to Andersson [2] and was used by Hörmander in [15], sect. 6 .

Definition 5.1. A homogeneous algebraic variety $V$ in $\mathbb{C}^{n}$ is said to be locally hyperbolic at $\xi_{0} \in V \cap \mathbb{R}^{n}$ if there exists a real, orthogonal choice of coordinates $(s, w)$ in $\mathbb{C}^{n}$ so that in these coordinates all the points $\left(\beta_{j}(w), w\right) \in V$ near $\xi_{0}$ are real when $w$ is real. We say that $V$ is locally hyperbolic at $\xi_{0}$ in these coordinates.

Remark. Hörmander [15], 6.5, shows that local hyperbolicity of $V$ at $\xi_{0}$ is a sufficient condition that $V$ satisfies $\operatorname{HPL}\left(\mathbb{R}^{n}\right.$, loc $)$ at $\xi_{0}$ and the converse holds if $V=V(P)$ is homogeneous of dimension 2 in $\mathbb{C}^{3}$, and $\xi_{0} \neq 0$.

Notation. Let $V$ be an algebraic variety in $\mathbb{C}^{n}$ of dimension $k$ and let $V_{h}$ denote its tangent cone at infinity. Assume that we have good coordinates for $V$ and $V_{h}$ according to 4.4. We define the "fiber distance" between $V$ and $V_{h}$ with respect to these coordinates as

$$
d(w):=\max _{1 \leq l \leq m}\left(\min _{1 \leq j \leq m^{\prime}}\left|\alpha_{l}(w)-\beta_{j}(w)\right|\right), \quad w \in \mathbb{C}^{k} .
$$

Obviously, we have $\operatorname{dist}\left(\left(\alpha_{l}(w), w\right), V_{h}\right) \leq d(w)$ for all $w \in \mathbb{C}^{k}$.

Lemma 5.2. Let $V$ be a pure dimensional algebraic variety of dimension $k$ in $\mathbb{C}^{n}$, let $V_{h}$ denote its tangent cone at infinity and let $\omega$ be a weight function. Assume that $W \cap\left(\mathbb{R}^{n} \backslash\{0\}\right) \neq \emptyset$ for each irreducible component $W$ of $V_{h}$ and that for each $\xi_{0} \in V_{h} \cap \mathbb{R}^{n}$ with $\left|\xi_{0}\right|=1$ there exist $\delta_{0}>0, R_{0} \geq 1$ and $M>0$ as well as good coordinates for $V$ and $V_{h}$ so that in these coordinates we have:

(1) $V_{h}$ is locally hyperbolic at $\xi_{0}$,

$(2) d(w) \leq M \omega(w)$ for all $w \in \pi\left(\left\{\zeta \xi \in \mathbb{C}^{n}:\left|\xi-\xi_{0}\right| \leq \delta,|\zeta| \geq R_{0}\right\}\right)$.

Then $V$ satisfies $\mathrm{PL}\left(\mathbb{R}^{n}, \omega\right)$. 
Proof. Evidently, the hypothesis implies that $V_{h}$ satisfies the dimension condition. Hence we get from [26], Thm. 5.1, that $V$ satisfies the following condition (RPL):

There exists $A_{1}>0$ so that for each $\rho>0$ there is $B_{1}>0$ such that each $u \in \operatorname{PSH}(V)$ satisfying $(\alpha)$ and $(\beta)$ also satisfies $(\gamma)$, where

( $\alpha) \quad u(z) \leq|z|+o(|z|), \quad z \in V$,

( $\beta) \quad u(z) \leq \rho|\operatorname{Im} z|, \quad z \in V$

$(\gamma) \quad u(z) \leq A_{1}|z|+B_{1}, \quad z \in V$.

Next note that $V_{h}$ satisfies $\operatorname{HPL}\left(\mathbb{R}^{n}\right.$, loc $)$ at each $\xi \in V_{h} \cap \mathbb{R}^{n}$ with $|\xi|=1$. This follows from the local Phragmén-Lindelöf condition as in the proof of (7) below. Hence Theorem 3.13 implies that $V_{h}$ satisfies $\operatorname{PL}\left(\mathbb{R}^{n}\right)$.

To prove that $V$ satisfies $\operatorname{PL}\left(\mathbb{R}^{n}, \omega\right)$ we fix $u \in \operatorname{PSH}(V)$ and assume that $u$ satisfies (1g) and $(2 \mathrm{~g})$ of Definition 2.5 for some $\rho>0$. To find constants $A>0$, depending only on $V$, and $B>0$, depending only on $V$ and $\rho$ such that $u$ satisfies $2.5(3 \mathrm{~g})$, we fix $\xi_{0} \in V_{h} \cap \mathbb{R}^{n}$ with $\left|\xi_{0}\right|=1$. By hypothesis we can choose good coordinates for $V$ and $V_{h}$ according to 4.4 so that $V_{h}$ is locally hyperbolic at $\xi_{0}$ in these coordinates. Therefore, we can choose $0<\delta<\delta_{0}$ and $\varepsilon>0$ so small that for a suitable labeling of the generic branches of $V_{h}$ in the present coordinates we find $\nu$ with $1<\nu \leq m^{\prime}$ so that

$$
\begin{aligned}
\left|\beta_{j}(w)-s_{0}\right|<\varepsilon \quad \text { for } \quad w \in U_{\delta}\left(w_{0}\right), 1 \leq j \leq \nu, \\
\left|\beta_{k}(w)-s_{0}\right| \geq 3 \varepsilon \quad \text { for } \quad w \in U_{\delta}\left(w_{0}\right), \nu<k \leq m^{\prime} .
\end{aligned}
$$

This implies that for $1 \leq j \leq \nu<k<m^{\prime}$ and all $w \in U_{\delta}\left(w_{0}\right)$ we have

$$
\left|\beta_{k}(\zeta w)-\beta_{j}(\zeta w)\right| \geq 2 \varepsilon|\zeta| \text { for all }|\zeta| \geq 1 \text {. }
$$

Label the branches $\left(\alpha_{l}(w), w\right)_{1 \leq l \leq m}$ of $V$ in such a way that $\left(\alpha_{l}(w), w\right)$ is tangent at infinity to some branch $\left(\beta_{j}(w), w\right)$ with $1 \leq j \leq \nu$ for $1 \leq l \leq \mu$ and to some branch $\left(\beta_{k}(w), w\right)$ with $\nu<k \leq m^{\prime}$ for $\mu<l \leq m$. Note that by 4.4(3) there are $0 \leq \sigma<1, D>0$ and $R_{1}>R_{0}$ so that for each $l$ with $1 \leq l \leq \mu$ there is $j$ with $1 \leq j \leq \nu$ so that for all $w \in U_{\delta}\left(w_{0}\right)$ we have

$$
\left|\alpha_{l}(\zeta w)-\beta_{j}(\zeta w)\right| \leq D|\zeta|^{\sigma},|\zeta| \geq R_{1} .
$$

From (4) and 4.4(3),

$$
\left|\alpha_{l}(\zeta w)-\beta_{j}(\zeta w)\right| \geq \varepsilon|\zeta|,|\zeta| \geq R_{1}, \text { all } 1 \leq l \leq \mu, \nu<j \leq m^{\prime} .
$$

Next, for $t>0$ and $R>0$ let

$$
\Gamma\left(\xi_{0}, t, R\right):=\left\{\zeta(s, w) \in \mathbb{C}^{n}:|\zeta|>R,\left|s-s_{0}\right|<\varepsilon,\left|w-w_{0}\right|<t\right\} .
$$

Then fix $R>R_{1}$ and define the function $v$ on $V_{h} \cap \Gamma\left(\xi_{0}, \delta / 2, R\right)$ by

$$
v\left(\beta_{j}(w), w\right):=\max \left\{u\left(\alpha_{l}(w), w\right):\left(\alpha_{l}(w), w\right) \in \Gamma\left(\xi_{0}, \delta / 2, R\right)\right\} .
$$

If $R$ is sufficiently large, then (5) implies that $v$ is well-defined. Moreover, $v$ is psh outside an analytic set and hence psh by Hörmander [15], 4.4. To estimate $v$, we fix $1 \leq l \leq \mu$. Then (5) together with (6) implies

$$
\min _{1 \leq j \leq m^{\prime}}\left|\alpha_{l}(\zeta w)-\beta_{j}(\zeta w)\right|=\min _{1 \leq j \leq \nu}\left|\alpha_{l}(\zeta w)-\beta_{j}(\zeta w)\right| .
$$

Hence we can choose $j=j(l)$ so that the minimum is attained at $j(l)$. We can assume that $\delta>0$ was chosen at the beginning so small that for each $1 \leq j \leq \nu$ the function $\beta_{j}(w)$ is real for $w$ real and $w \in U_{\delta}\left(w_{0}\right)$. Hence we can define

$$
\varphi: U_{\delta}\left(w_{0}\right) \rightarrow\left[0, \infty\left[, \varphi(w):=\max \left\{\left|\operatorname{Im} \beta_{j}(w)\right|: 1 \leq j \leq \nu\right\} .\right.\right.
$$


Then $\varphi$ is bounded by (3) and psh outside an analytic subset of $U_{\delta}\left(w_{0}\right)$. Therefore, $\varphi$ is psh by Hörmander [15], 4.4. Hence the local Phragmén-Lindelöf principle 3.16 implies the existence of $C_{0}=C_{0}\left(\xi_{0}\right) \geq 1$ such that

$$
\left|\operatorname{Im} \beta_{j}(w)\right| \leq \varphi(w) \leq C_{0}|\operatorname{Im} w| \text { for } 1 \leq j \leq \nu,\left|w-w_{0}\right| \leq \delta / 2 .
$$

Consequently, for $1 \leq l \leq \mu$ and $j=j(l)$ :

$$
\begin{aligned}
\left|\operatorname{Im}\left(\alpha_{l}(\zeta w), \zeta w\right)\right| & \leq\left|\operatorname{Im}\left(\alpha_{l}(\zeta w)-\beta_{j}(\zeta w)\right)\right|+\left|\operatorname{Im}\left(\beta_{j}(\zeta w), \zeta w\right)\right| \\
& \leq d(\zeta w)+\left(C_{0}+1\right)|\operatorname{Im} \zeta w| .
\end{aligned}
$$

Next note that for $l$ with $\mu<l \leq m$ there is $j$ with $\nu<j \leq m^{\prime}$ so that (5) holds. Hence (4) implies that $\left(\alpha_{l}(\zeta w), \zeta w\right) \notin \Gamma\left(\xi_{0}, \delta / 2, R\right)$ if $R$ is sufficiently large enough. Therefore the hypothesis together with (8) and the estimates on $u$ imply that $v$ satisfies

$$
\begin{aligned}
& v\left(\beta_{j}(w), w\right) \leq\left(C_{0}+1\right)|\operatorname{Im} w|+O(\omega(w)),\left(\beta_{j}(w), w\right) \in \Gamma\left(\xi_{0}, \delta / 2, R\right), \\
& v\left(\beta_{j}(w), w\right) \leq \rho\left(C_{0}+1\right)|\operatorname{Im} w|+\rho d(w),\left(\beta_{j}(w), w\right) \in \Gamma\left(\xi_{0}, \delta / 2, R\right) .
\end{aligned}
$$

Since $u$ satisfies $2.5(1 \mathrm{~g})$ and $(2 \mathrm{~g})$ with $\rho>0$ we deduce from (RPL) that

$$
u(z) \leq A_{1}|z|+B_{1} \text { for all } z \in V .
$$

From this, for $1 \leq l \leq \mu$ and a suitable $j=j(l)$ with $1 \leq j \leq \nu$ :

$$
\begin{aligned}
u\left(\alpha_{l}(w), w\right) & \leq A_{1}\left|\alpha_{l}(w)-\beta_{j}(w)\right|+A_{1}\left|\left(\beta_{j}(w), w\right)\right|+B_{1} \\
& \leq A_{1} d(w)+A_{1}\left|\left(\beta_{j}(w), w\right)\right|+B_{1} .
\end{aligned}
$$

Since $\omega(t)=o(t)$ and since it may be assumed that $C_{0} \geq C$, where $C$ is the constant in 4.4(2), we get from this the existence of a constant $B_{2}>B_{1}$ so that

$$
v\left(\beta_{j}(w), w\right) \leq A_{1}\left(C_{0}+2\right)|w|+B_{2} \text { for }\left(\beta_{j}(w), w\right) \in \Gamma\left(\xi_{0}, \delta / 2, R\right) .
$$

Choose $\alpha>0$ so that $5 \alpha<\min (\varepsilon, \delta / 2,1)$ and fix $z_{0} \in V_{h}$ satisfying $\left|\frac{z_{0}}{t}-\xi_{0}\right|<\alpha$ for some $t>R$. Then we let

$$
U:=\left\{z \in \mathbb{C}^{n}:\left|z-\operatorname{Re} z_{0}\right|<2 \alpha\left|z_{0}\right|\right\} .
$$

To show that $U \subset \Gamma\left(\xi_{0}, \frac{\delta}{2}, R\right)$, note that $\left|\xi_{0}\right|=1$ implies

$$
\left|z_{0}\right|=t|\xi| \leq t\left|\xi-\xi_{0}\right|+t\left|\xi_{0}\right|<t(\alpha+1) .
$$

Therefore, each $z \in U$ satisfies

$$
\left|\frac{z}{t}-\xi_{0}\right| \leq\left|\frac{z}{t}-\frac{\operatorname{Re} z_{0}}{t}\right|+\left|\frac{\operatorname{Re} z_{0}}{t}-\xi_{0}\right|<\frac{1}{t} 2 \alpha t(\alpha+1)+\alpha \leq 5 \alpha
$$

and consequently $z \in \Gamma\left(\xi_{0}, \frac{\delta}{2}, R\right)$.

Next let $H$ be the function in Lemma 3.4 and $c$ the constant in that lemma. Let $C_{1}:=\max \left\{(2 \alpha c)^{-1} A_{1}\left(C_{0}+2\right)(1+2 \alpha), C_{0}+1\right\}$ and define the function $\widetilde{v}$ on $U \cap V_{h}$ by

$$
\widetilde{v}(z):=\max \left\{v(z)+C_{1} 2 \alpha\left|z_{0}\right| H\left(\frac{z-\operatorname{Re} z_{0}}{2 \alpha\left|z_{0}\right|}\right), C_{1}\left|\operatorname{Im} z_{2}\right|+B_{2}+1\right\} .
$$

From (10) and Lemma 3.4, on $\partial U \cap V_{h}$ the first term in the definition of $v$ can be estimated by

$$
A_{1}\left(C_{0}+2\right)|z|+B_{2}+C_{1}|\operatorname{Im} z|-c C_{1} 2 \alpha\left|z_{0}\right| \leq C_{1}|\operatorname{Im} z|+B_{2} .
$$


Hence $\widetilde{v}$ can be extended to a psh function on $V_{h}$ by defining it as $C_{1}|\operatorname{Im} z|+B_{2}+1$ on $V_{h} \backslash U$. Since $U$ is contained in $\Gamma\left(\xi_{0}, \delta / 2, R\right)$, we get from (2), (9) and the definition of $\widetilde{v}$ that $\widetilde{v}$ satisfies the estimates

$$
\widetilde{v}(z) \leq C_{1}|\operatorname{Im} z|+O(1) \text { and } \widetilde{v}(z) \leq \rho C_{1}|\operatorname{Im} z|+\rho M \omega(z), z \in V_{h} .
$$

We have already noted that $V_{h}$ satisfies $\operatorname{PL}\left(\mathbb{R}^{n}\right)$. Hence Proposition 2.8 and Theorem 3.3 imply the existence of a constant $A_{2}$, depending only on $V_{h}$, and of a constant $B_{3}$, depending on $V_{h}, \rho, \xi_{0}$ and $M$, such that

$$
\widetilde{v}(z) \leq A_{2} C_{1}|\operatorname{Im} z|+B_{3} \omega(z) \text { for all } z \in V_{h} .
$$

Evaluating this inequality at $z_{0}$ and using 3.4(4), we get

$$
v\left(z_{0}\right) \leq A_{2} C_{1}\left|\operatorname{Im} z_{0}\right|+B_{3} \omega\left(z_{0}\right) .
$$

Since $z_{0} \in V_{h}$ was an arbitrary point in $t U_{\alpha}\left(\xi_{0}\right)$ for some $t>R$, the estimate (11) holds for all $z_{0} \in V_{h} \cap \bigcup_{t>R} t U_{\alpha}\left(\xi_{0}\right)$.

Next observe that $\xi_{0}$ was an arbitrary point of the set $L:=\left\{\xi \in V_{h} \cap \mathbb{R}^{n}:|\xi|=1\right\}$ and that $\alpha=\alpha\left(\xi_{0}\right)$ and $R=R\left(\xi_{0}\right)$. Therefore we can cover the compact set $L$ by finitely many of these balls $U_{j}=U_{\alpha\left(\xi_{j}\right)}\left(\xi_{j}\right), 1 \leq j \leq p$. This, together with 4.4(3) and a standard compactness argument implies the existence of $R_{0} \geq 1$ and $M>0$ such that

$$
|z| \leq M|\operatorname{Im} z| \text { for all } z \in V \backslash\left(\bigcup_{j=1}^{p} \bigcup_{t \geq R_{0}} t U_{j}\right), \quad|z| \geq R_{0}
$$

and the existence of $A_{0} \geq 1$ and $B_{0}>0, A_{0}$ depending only on $V, B_{0}$ depending only on $V$ and $\rho$, such that

$$
u(z) \leq A_{0}|\operatorname{Im} z|+B_{0} \omega(z) \text { for all } z \in V \cap\left(\bigcup_{j=1}^{p} \bigcup_{t \geq R_{0}} t U_{j}\right), \quad|z| \geq R_{0} .
$$

Now (12) and (13) in connection with (RPL) give

$$
u(z) \leq \max \left(A_{0}, M A_{1}\right)|\operatorname{Im} z|+B_{0} \omega(z)+A_{1} R_{0}+B_{1} \text { for all } z \in V .
$$

Obviously, this implies that $u$ satisfies $(3 \mathrm{~g})$ in 2.5. Hence $V$ satisfies $\operatorname{PL}\left(\mathbb{R}^{n}, \omega\right)$.

Lemma 5.3. Let $V$ be a pure dimensional algebraic variety in $\mathbb{C}^{n}$ of dimension $k$ and let $V_{h}$ denote its tangent cone at infinity. Let $W_{1}, \ldots, W_{p}$ be the irreducible components of $V_{h}$, let $a_{i}$ be the number that corresponds to $W_{i}$ for $1 \leq i \leq p$ by Lemma 4.5, and let $a:=\max _{1 \leq i \leq p} a_{i}$. If $V_{h} \backslash\{0\}$ is a manifold, then

$$
\operatorname{dist}\left(z, V_{h}\right)=O\left(|z|^{a}\right) \text { for } z \in V,|z| \rightarrow \infty .
$$

Proof. For each point $\xi_{0} \in V_{h},\left|\xi_{0}\right|>0$, by hypothesis, we can choose good coordinates in $\mathbb{C}^{n}$ for $V$ and $V_{h}$ so that the cover $\pi: V_{h} \rightarrow \mathbb{C}^{k}$ is unbranched over $w_{0}$, where $\xi_{0}=\left(s_{0}, w_{0}\right)$. We assume $\left|w_{0}\right|=1$. As in the proof of Lemma 4.5, choose $\delta>0$ and $R>1$ so that $\pi: V_{h} \rightarrow \mathbb{C}^{k}$ is an analytic cover over the set

$$
\Gamma\left(w_{0}, \delta, R\right):=\left\{\zeta w \in \mathbb{C}^{k}:\left|w-w_{0}\right|<\delta,|\zeta|>R\right\} .
$$

Fix a branch $(\beta(w), w)$ of $V_{h}$ with $\xi_{0}=\left(\beta\left(w_{0}\right), w_{0}\right)$ and define for $\left|w-w_{0}\right|<\delta$ and $|\zeta|>R$ the function

$$
\begin{aligned}
& v(\zeta, w):=\max \left\{\log \left|\alpha_{l}(\zeta w)-\zeta \beta(w)\right|:\right. \\
&\left.\left|\alpha_{l}(\zeta w)-\zeta \beta(w)\right| \leq C(1+|\zeta w|)^{1-\varepsilon}\right\},
\end{aligned}
$$


$C$ and $\varepsilon$ being chosen according to 4.4 . Then $v$ is psh in $(\zeta, w)$. Next define

$$
u(w):=\limsup _{|\zeta| \rightarrow \infty} \frac{v(\zeta, w)}{\log |\zeta|},\left|w-w_{0}\right|<\delta .
$$

If $\xi_{0}$ belongs to $W_{i}$, then estimate (6) in Lemma 4.5 implies $u(w) \leq a_{i}$ for all $\left|w-w_{0}\right|<\delta$. Therefore we get from Hartogs' lemma (see e.g. [14], Thm. 1.6.13) that for each $\eta>0$ there exists $R_{\eta}>0$ so that

$$
v(\zeta, w) \leq a_{i}+\eta \text { for }|\zeta| \geq R_{\eta} \text { and }\left|w-w_{0}\right| \leq \delta / 2 .
$$

This implies

$$
v(\zeta, w) \leq\left(a_{i}+\eta\right) \log ^{+}|\zeta| \text { for }|\zeta| \geq R_{\eta},\left|w-w_{0}\right| \leq \delta / 2 .
$$

Now let

$$
C_{1}:=\sup \left\{\log \left|\alpha_{l}(\zeta w)-\zeta \beta(w)\right|:|w|=1, \zeta=R \text {, all } l\right\} .
$$

Since $\zeta \mapsto v(\zeta, w)$ is subharmonic, the maximum principle implies

$$
v(\zeta, w) \leq\left(a_{i}+\eta\right) \log ^{+}|\zeta|+C_{1} \text { for }|\zeta| \geq R,\left|w-w_{0}\right| \leq \delta / 2,|w|=1 .
$$

Since $\eta>0$ was chosen arbitrarily, it follows that

$$
v(\zeta, w) \leq a_{i} \log ^{+}|\zeta|+C_{1} \text { for }|\zeta| \geq R,\left|w-w_{0}\right| \leq \delta / 2,|w|=1 .
$$

From this we get

$$
\operatorname{dist}\left(z, V_{h}\right) \leq C_{1}|z|^{a_{i}}
$$

for all $z \in V, z=\left(\alpha_{l}(\zeta w), \zeta w\right)$ satisfying $|\zeta| \geq R$ and $\left|\alpha_{l}(\zeta w)-\zeta \beta(w)\right| \leq$ $C(1+|\zeta w|)^{1-\varepsilon}$ for some $w \in \mathbb{C}^{k}$ with $|w|=1$ and $\left|w-w_{0}\right|<\delta / 2$. Since all points in $V$ outside the ball of radius $R$ around zero can be reached this way, the result follows by a compactness argument.

Proposition 5.4. Let $V$ be a pure dimensional algebraic variety in $\mathbb{C}^{n}$ and let $V_{h}$ be its tangent cone at infinity. Assume that for each irreducible component $W$ of $V$ the tangent cone $W_{h}$ at infinity is a manifold outside the origin. If $V$ satisfies $\operatorname{PL}\left(\mathbb{R}^{n}, \omega\right)$ for some weight function $\omega$, then

$$
\operatorname{dist}\left(z, V_{h}\right)=O(\omega(z)), z \in V,|z| \rightarrow \infty .
$$

In particular, if $\omega(t)=o\left(t^{\varepsilon}\right)$ for each $\varepsilon>0$, then

$$
\operatorname{dist}\left(z, V_{h}\right)=O(1), z \in V,|z| \rightarrow \infty \text {. }
$$

Proof. Let $V_{1}, \ldots, V_{q}$ be the irreducible components of $V$ and denote by $\left.a_{j} \in\right]-\infty, 1[$ the number that is attached to $V_{j}$ by Lemma 5.3. Then this lemma shows

$$
\operatorname{dist}\left(z, V_{j, h}\right) \leq O\left(|z|^{a_{j}}\right), z \in V_{j},|z| \rightarrow \infty .
$$

Without restriction we may assume $a_{1}=\max _{1 \leq j \leq q} a_{j}$. Since $V$ satisfies the condition $\operatorname{PL}\left(\mathbb{R}^{n}, \omega\right), V_{1}$ also satisfies this condition by Proposition 2.6. Hence Proposition 4.8 implies

$$
|t|^{a_{1}}=O(\omega(t)) \text { as } t \mapsto \infty .
$$

Obviously, the result follows from this and (1).

If $\omega$ satisfies $\omega(t)=o\left(t^{\varepsilon}\right)$ for each $\varepsilon>0$, then (2) implies $a_{1} \leq 0$ and hence $a_{j} \leq 0$ for $1 \leq j \leq q$. Thus (1) implies $\operatorname{dist}\left(z, V_{h}\right)=O(1)$ for $z \in V$ and $|z| \rightarrow \infty$. 
Remark. Note that the assertion of Proposition 5.4 may fail if for some irreducible component of $V$ the tangent cone at infinity has singularities outside the origin. This follows from Example 4.9, since $V\left(P_{1}\right)$ satisfies the condition $\mathrm{PL}\left(\mathbb{R}^{3}, \log (2+t)\right)$ by $4.9(2)$, while $\operatorname{dist}\left(z, V\left(P_{1}\right)_{h}\right)$ is unbounded by $4.9(5)$.

Remark 5.5. Note that Proposition 5.4 applies to all varieties $V$ of dimension 1. In particular, it follows that the second statement in [21], 4.10, holds for $n=2$. By the preceding remark it does not hold for $n>2$. However, only the case $n=2$ was used in [21] to derive [21], 4.11, from [21], 4.10.

Theorem 5.6. Let $V$ be a pure dimensional algebraic variety in $\mathbb{C}^{n}$, let $V_{h}$ denote its tangent cone at infinity and assume that $V_{h} \backslash\{0\}$ is a manifold. Then for each weight function $\omega$ the following assertions are equivalent:

(1) $V$ satisfies $\mathrm{PL}\left(\mathbb{R}^{n}, \omega\right)$.

(2) $V_{h}$ satisfies $\operatorname{PL}\left(\mathbb{R}^{n}\right)$ and $\operatorname{dist}\left(z, V_{h}\right)=O(\omega(z)), z \in V,|z| \rightarrow \infty$.

(3) $V_{h}$ satisfies the strong dimension condition and $\operatorname{dist}\left(z, V_{h}\right)=O(\omega(z)), z \in$ $V,|z| \rightarrow \infty$.

Proof. (1) $\Rightarrow(2)$ : Theorem 4.1 implies that $V_{h}$ satisfies $\mathrm{PL}\left(\mathbb{R}^{n}\right)$ and Proposition 5.4 implies that the second condition in (2) holds.

$(2) \Rightarrow(3)$ : This is a consequence of Theorem 3.13.

$(3) \Rightarrow(1)$ : In order to apply Lemma 5.2 , let $W$ be an irreducible component of $V_{h}$. If we assume that $W \cap \mathbb{R}^{n}=\{0\}$, then $V_{h}$ does not satisfy the dimension condition at zero. Hence the hypothesis implies that $W \cap\left(\mathbb{R}^{n} \backslash\{0\}\right) \neq \emptyset$. Next fix $\xi_{0} \in V_{h} \cap \mathbb{R}^{n}$ with $\left|\xi_{0}\right|=1$ and choose good coordinates for $V$ and $V_{h}$ according to 4.4. Since $V_{h} \backslash\{0\}$ is a manifold, we can choose them in such a way that the covering map $\pi: V_{h} \rightarrow \mathbb{C}^{k}$ is unbranched over a neighborhood $U$ of $w_{0}$ in $\mathbb{C}^{k}$, where $\xi_{0}=\left(s_{0}, w_{0}\right)$. Since $V_{h}$ satisfies the dimension condition at $\xi_{0}, V_{h}$ is locally hyperbolic at $\xi_{0}$ in these coordinates. Hence condition 5.2(1) is fulfilled.

To show that also condition 5.2(2) is satisfied, note that by hypothesis there exists $L_{0}>0$ with

$$
\operatorname{dist}\left(z, V_{h}\right) \leq L_{0} \omega(z) \quad \text { for }|z| \geq 3, z \in V .
$$

Fix $l$ with $1 \leq l \leq m, w \in U$ and $\zeta \in \mathbb{C},|\zeta|$ large. Then, for some $\varepsilon>0$, the points $\left\{\beta_{j}(\zeta w)\right\}_{j=1}^{m^{\prime}}$ are of distance at least $\varepsilon|\zeta w|$ apart. Hence 4.4(3) implies that to each $\alpha_{l}(\zeta w)$ there corresponds a unique $j=j(l)$ such that $\alpha_{l}(\zeta w)$ is closer to $\beta_{j}(\zeta w)$ than to any other $\beta_{\nu}(\zeta w)$. Therefore, the point in $V_{h}$ closest to $\left(\alpha_{l}(\zeta w), \zeta w\right)$ has the form $\left(\beta_{j}(\zeta w+\Delta(\zeta w)), \zeta w+\Delta(\zeta w)\right)$. Since $\operatorname{dist}\left(\left(\alpha_{l}(\zeta w), \zeta w\right), V_{h}\right) \leq L w(\zeta w)$ for some constant $L \geq L_{0}$, we must have $|\Delta(\zeta w)| \leq L \omega(\zeta w)$ if $|\zeta|$ is sufficiently large. This implies

$$
\begin{aligned}
\left|\alpha_{l}(\zeta w)-\beta_{j}(\zeta w)\right| \leq & \left|\alpha_{l}(\zeta w)-\beta_{j}(\zeta w+\Delta(\zeta w))\right| \\
& +\left|\beta_{j}(\zeta w+\Delta(\zeta w))-\beta_{j}(\zeta w)\right| \\
\leq & L \omega(\zeta w)+M L \omega(\zeta w)
\end{aligned}
$$

since we can assume (by shrinking $U$ if necessary) that

$$
\left|\beta_{\nu}(w+h)-\beta_{\nu}(w)\right| \leq M|h|
$$

for all $w \in W$ and $1 \leq \nu \leq m^{\prime}$. Since $1 \leq l \leq m$ was chosen arbitrarily, this implies that condition 5.2(2) is fulfilled. Hence (1) follows from Lemma 5.2. 
To derive a useful corollary from Theorem 5.6, we will apply the following lemma.

Lemma 5.7. Let $P$ and $Q$ be complex polynomials in $n$ variables with $\operatorname{deg} Q<$ $\operatorname{deg} P$ and let $\delta:[0, \infty[\rightarrow] 0, \infty[$ be increasing. Then the following conditions are equivalent:

(1) $\operatorname{dist}(z, V(P))=O(\delta(|z|))$ for $z \in V(P+Q), \quad|z| \rightarrow \infty$.

(2) There exists $D \geq 1$ such that

$$
|Q(z)| \leq D \max _{|\alpha|>0}\left|P^{(\alpha)}(z)\right| \delta(|z|)^{|\alpha|} \text { for all } z \in V(P+Q) .
$$

Proof. By the proof of Hörmander [13], Lemma 4.1.1, there exists a constant $C \geq 1$ such that for each polynomial $R \in \mathbb{C}\left[z_{1}, \ldots, z_{n}\right]$ with $\operatorname{deg} R \leq \operatorname{deg} P$ we have

$$
C^{-1} \leq \operatorname{dist}(z, V(R)) \sum_{|\alpha|>0}\left|\frac{R^{(\alpha)}(z)}{R(z)}\right|^{1 /|\alpha|} \leq C, \quad z \in \mathbb{C}^{n} \backslash V(R) .
$$

Now assume that (1) holds and apply (3) to $P$ and $z \in V(P+Q) \backslash V(P)$. Then $P(z)=-Q(z)$ and (1) imply the existence of some $L \geq 1$ such that

$$
1 \leq L \delta(|z|) \sum_{|\alpha|>0}\left|\frac{P^{(\alpha)}(z)}{P(z)}\right|^{1 /|\alpha|}=L \sum_{|\alpha|>0}\left|\delta(|z|)^{|\alpha|} \frac{P^{(\alpha)}(z)}{Q(z)}\right|^{1 /|\alpha|} .
$$

Estimating the (finite) sum by a maximum, this implies (2).

If (2) holds, then there exists $M \geq 1$ such that for $z \in V(P+Q) \backslash V(P)$ we have

$$
1 \leq M \max _{|\alpha|>0}\left|\frac{P^{(\alpha)}(z)}{Q(z)}\right|^{1 /|\alpha|} \delta(|z|) \leq M \delta(|z|) \sum_{|\alpha|>0}\left|\frac{P^{(\alpha)}(z)}{P(z)}\right|^{1 /|\alpha|} .
$$

By (3), this implies (1).

Corollary 5.8. Let $P_{m}$ be a homogeneous polynomial of degree $m$ in $n$ variables which satisfies grad $P_{m}(z) \neq 0$ for all $z \in \mathbb{C}^{n} \backslash\{0\}$. Then the following assertions are equivalent:

(1) $V\left(P_{m}\right)$ satisfies $\mathrm{PL}\left(\mathbb{R}^{n}\right)$.

(2) $V\left(P_{m}\right)$ satisfies the strong dimension condition.

(3) There exist $k \in \mathbb{N}, Q \in \mathbb{C}\left[z_{1}, \ldots, z_{n}\right]$ with $\operatorname{deg} Q<k m$, and a weight function $\omega$ so that $V\left(P_{m}^{k}+Q\right)$ satisfies $\mathrm{PL}\left(\mathbb{R}^{n}, \omega\right)$.

(4) For each $k \in \mathbb{N}$ and each $Q \in \mathbb{C}\left[z_{1}, \ldots, z_{n}\right]$ with $\operatorname{deg} Q=l<k m$ we have:

(a) If $l \leq k(m-1)$, then $V\left(P_{m}^{k}+Q\right)$ satisfies $\operatorname{PL}\left(\mathbb{R}^{n}, \log (2+t)\right)$.

(b) If $l>k(m-1)$, then $V\left(P_{m}^{k}+Q\right)$ satisfies $\mathrm{PL}\left(\mathbb{R}^{n}, 1+t^{\beta}\right)$ for $\beta=1+\frac{l}{k}-m$.

Proof. (1) $\Rightarrow(4)$ : Fix $k \in \mathbb{N}$ and note that $V\left(P_{m}^{k}\right)=V\left(P_{m}\right)$ is a manifold outside the origin by hypothesis. Therefore, (a) and (b) follow from Theorem 5.6, if we prove the corresponding estimates for $\operatorname{dist}\left(z, V\left(P_{m}\right)\right), z \in V\left(P_{m}^{k}+Q\right),|z| \rightarrow \infty$. To do this, note that

$$
\operatorname{grad} P_{m}^{k}(z)=k P_{m}^{k-1}(z) \operatorname{grad} P_{m}(z) \text { for all } z \in \mathbb{C}^{n} .
$$

Hence the hypothesis implies

$$
\max _{|\alpha|=1}\left|\left(P_{m}^{k}\right)^{(\alpha)}(z)\right|>0 \text { for all } z \in \mathbb{C}^{n} \backslash V\left(P_{m}\right) .
$$


For $z \in V\left(P_{m}\right)$ the hypothesis gives that for some $j$ with $1 \leq j \leq n$ we have $\frac{\partial P_{m}}{\partial z_{j}}(z) \neq 0$ and hence

$$
\left(\frac{\partial}{\partial z_{j}}\right)^{k} P_{m}^{k}(z)=k !\left(\frac{\partial P_{m}}{\partial z_{j}}(z)\right)^{k} \neq 0 .
$$

Consequently, we have

$$
\max _{0<|\alpha| \leq k}\left|\left(P_{m}^{k}\right)^{(\alpha)}(z)\right|>0 \text { for all } z \in \mathbb{C}^{n}, \quad|z|=1 .
$$

Now note that $\left(P_{m}^{k}\right)^{(\alpha)}$ is homogeneous of degree $k m-|\alpha|$ for each $\alpha \in \mathbb{N}_{0}^{n}$ with $|\alpha| \leq k m$. Hence, by (5), there exists $\delta>0$ such that

$$
\max _{0<|\alpha| \leq k}\left|\left(P_{m}^{k}\right)^{(\alpha)}(z)\right| \geq \delta|z|^{k(m-1)} \quad \text { for all } z \in \mathbb{C}^{n}, \quad|z| \geq 1 .
$$

If $\operatorname{deg} Q=l \leq k(m-1)$, this implies the existence of $D \geq 1$ such that

$$
|Q(z)| \leq D \max _{|\alpha|>0}\left|\left(P_{m}^{k}\right)^{(\alpha)}(z)\right| \text { for all } z \in \mathbb{C}^{n} .
$$

Applying Lemma 5.7 with $\delta \equiv 1$, we get

$$
\operatorname{dist}\left(z, V\left(P_{m}\right)\right)=O(1) \text { for } z \in V\left(P_{m}^{k}+Q\right), \quad|z| \rightarrow \infty .
$$

Hence (a) follows from Theorem 5.6.

If $k(m-1)<l<k m$ and $\beta=1+\frac{l}{k}-m$, then (5) implies the existence of $\delta>0$ such that

$$
\max _{0<|\alpha| \leq k}\left|\left(P_{m}^{k}\right)^{(\alpha)}(z)\right||z|^{\beta|\alpha|} \geq \delta|z|^{k(m-1)-\beta k}=\delta|z|^{l} \text { for all } z \in \mathbb{C}^{n},|z| \geq 1 .
$$

Hence for each $Q \in \mathbb{C}\left[z_{1}, \ldots, z_{n}\right]$ with $\operatorname{deg} Q=l$ there exists $D \geq 1$ such that

$$
|Q(z)| \leq D \max _{0<|\alpha|}\left|\left(P_{m}^{k}\right)^{(\alpha)}(z)\right|\left(1+|z|^{\beta}\right)^{|\alpha|} \text { for all } z \in \mathbb{C}^{n} .
$$

Applying Lemma 5.7 with $\delta(t)=1+t^{\beta}$, we get

$$
\operatorname{dist}\left(z, V\left(P_{m}\right)\right)=O\left(|z|^{\beta}\right) \text { for } z \in V\left(P_{m}^{k}+Q\right), \quad|z| \rightarrow \infty .
$$

Hence (b) follows from Theorem 5.6.

$(4) \Rightarrow(3)$ : This is obvious.

(3) $\Rightarrow(2)$ : This holds by Theorem 4.1 .

$(2) \Rightarrow(1)$ : This holds by Corollary 3.17 .

Example 5.9. For $n \geq 3$ and $m \geq 2$ let $P_{m} \in \mathbb{C}\left[z_{1}, \ldots, z_{n}\right]$ be of the form

$$
P_{m}\left(z_{1}, \ldots, z_{n}\right)=\sum_{j=1}^{n} a_{j} z_{j}^{m},
$$

where $a_{j} \in \mathbb{R} \backslash\{0\}$ for $1 \leq j \leq n$. Then the variety $V(P)$ of the polynomial $P:=P_{m}+Q$ satisfies $\operatorname{PL}\left(\mathbb{R}^{n}, \log \right)$ for some/all $Q \in \mathbb{C}\left[z_{1}, \ldots, z_{n}\right]$ with $\operatorname{deg} Q<m$ if and only if either $m$ is odd or $m$ is even and there are $j, k$ so that sign $a_{j} \neq \operatorname{sign}$ $a_{k}$. This follows from Corollary 5.8 and [21], 4.9, where it was shown that all the polynomials $P_{m}$ are irreducible and that $V\left(P_{m}\right)$ satisfies $\mathrm{PL}\left(\mathbb{R}^{n}, \log \right)$ if and only if the given conditions hold. 


\section{The Phragmén-Lindelöf Condition PL $\{\Omega, \omega\}$}

In this section we introduce a further Phragmén-Lindelöf condition for algebraic varieties in $\mathbb{C}^{n}$, called $\operatorname{PL}\{\Omega, \omega\}$. By the results of $[23]$, sect. 5 , the variety $V(P)$ of a polynomial $P \in \mathbb{C}\left[z_{1}, \ldots, z_{n}\right]$ satisfies the condition $\operatorname{PL}\{\Omega, \omega\}$ for a convex open set $\Omega$ in $\mathbb{R}^{n}$ if and only if the differential operator $P(D)$ acting on the $\{\omega\}-$ ultradifferentiable functions $\mathcal{E}_{\{\omega\}}(\Omega)$ or on the $\{\omega\}$-ultradistributions $\mathcal{D}_{\{\omega\}}^{\prime}(\Omega)$ admits a continuous linear right inverse. We show that the main results for $\operatorname{PL}(\Omega, \omega)$ have corresponding counterparts for $\operatorname{PL}\{\Omega, \omega\}$ and can be derived easily from these.

Throughout this section $\omega$ will always denote a weight function that satisfies $\log (t)=o(\omega(t))$ as $t$ tends to infinity. For such weight functions $\omega$ we let

$$
S_{\omega}:=\{\sigma: \sigma \text { is a weight function satisfying } \log =o(\sigma) \text { and } \sigma=o(\omega)\} .
$$

Definition 6.1. Let $V$ be an algebraic variety in $\mathbb{C}^{n}$, let $\Omega$ be a convex open set in $\mathbb{R}^{n}$ and let $\omega$ be as above.

(a) $V$ satisfies the condition $\operatorname{PL}\{\Omega, \omega\}$ if the following holds: For each $K \in \mathcal{K}(\Omega)$ there exists $K^{\prime} \in \mathcal{K}(\Omega)$ such that for each $K^{\prime \prime} \in \mathcal{K}(\Omega)$ there exists $\sigma \in S_{\omega}$ such that each $u \in \operatorname{PSH}(V)$ satisfying (1) and (2) also satisfies (3), where

(1) $u(z) \leq h_{K}(\operatorname{Im} z)+o(\omega(z)), \quad z \in V$,

(2) $u(z) \leq h_{K^{\prime \prime}}(\operatorname{Im} z), \quad z \in V$,

(3) $u(z) \leq h_{K^{\prime}}(\operatorname{Im} z)+\sigma(z), \quad z \in V$.

(b) $V$ satisfies the condition $\widetilde{\mathrm{PL}}\{\Omega, \omega\}$ if the following holds: For each $K \in \mathcal{K}(\Omega)$ there exists $K^{\prime} \in \mathcal{K}(\Omega)$ such that for each $K^{\prime \prime} \in \mathcal{K}(\Omega)$ and each $l \in \mathbb{N}$ there exist $m \in \mathbb{N}$ and $C>0$ such that each $u \in \operatorname{PSH}(V)$ satisfying (1) and (2) also satisfies (3), where

(1) $u(z) \leq h_{K}(\operatorname{Im} z)+o(\omega(z)), \quad z \in V$,

(2) $u(z) \leq h_{K^{\prime \prime}}(\operatorname{Im} z)+\frac{1}{m} \omega(z), \quad z \in V$,

(3) $u(z) \leq h_{K^{\prime}}(\operatorname{Im} z)+\frac{1}{l} \omega(z), \quad z \in V$.

The following proposition shows that the conditions $\operatorname{PL}\{\Omega, \omega\}$ and $\widetilde{\mathrm{PL}}\{\Omega, \omega\}$ are equivalent and are closely related to the Phragmén-Lindelöf conditions $\operatorname{PL}(\Omega, \kappa)$, that we have investigated in the previous sections.

Proposition 6.2. Let $V$ be an algebraic variety in $\mathbb{C}^{n}$ and $\Omega$ a convex open subset in $\mathbb{R}^{n}$. Then the following assertions are equivalent:

(1) $V$ satisfies $\operatorname{PL}\{\Omega, \omega\}$.

(2) $V$ satisfies $\widetilde{\mathrm{PL}}\{\Omega, \omega\}$.

(3) $V$ satisfies $\mathrm{PL}(\Omega, \kappa)$ for some $\kappa \in S_{\omega}$.

Proof. (1) $\Rightarrow(3)$ : If $K \in \mathcal{K}(\Omega)$ is given, we choose $K^{\prime} \in \mathcal{K}(\Omega)$ according to $\operatorname{PL}\{\Omega, \omega\}$. Then fix a sequence $\left(K_{j}\right)_{j \in \mathbb{N}}$ in $\mathcal{K}(\Omega)$ such that for each $K^{\prime \prime} \in \mathcal{K}(\Omega)$ there is $j \in \mathbb{N}$ with $K^{\prime \prime} \subset K_{j}$ and apply $\operatorname{PL}\{\Omega, \omega\}$ to $K_{j}$ to get $\sigma_{j} \in S_{\omega}$. By Braun, Meise and Taylor [6], 1.9, there exists $\kappa \in S_{\omega}$ satisfying $\kappa \geq 1$ and $\sigma_{j}=o(\kappa)$ for each $j \in \mathbb{N}$. To show that $V$ satisfies $\operatorname{PL}(\Omega, \kappa)$, we fix $u \in \operatorname{PSH}(V)$ satisfying

$$
u(z) \leq h_{K}(\operatorname{Im} z)+O(\kappa(z)) \text { and } u(z) \leq h_{K^{\prime \prime}}(\operatorname{Im} z) .
$$

Then choose $j \in \mathbb{N}$ with $K^{\prime \prime} \subset K_{j}$ and note that $u$ satisfies (1) and (2) of PL $\{\Omega, \omega\}$ with $K^{\prime \prime}$ replaced by $K_{j}$. Since $V$ satisfies $\operatorname{PL}\{\Omega, \omega\}$, our choice of $\sigma_{j}$ and $\kappa$ implies: $u(z) \leq h_{K^{\prime}}(\operatorname{Im} z)+\sigma_{j}(z) \leq h_{K^{\prime}}(\operatorname{Im} z)+\kappa(z)+C_{j} \leq h_{K^{\prime}}(\operatorname{Im} z)+C_{j} \kappa(z), z \in V$. Hence $V$ satisfies $\operatorname{PL}(\Omega, \kappa)$. 
$(3) \Rightarrow(2)$ : Without loss of generality we may assume that $\Omega$ contains the closed unit ball $B_{1}(0)$ of $\mathbb{R}^{n}$. Then it suffices to consider only those $K \in \mathcal{K}(\Omega)$ with $B_{1}(0) \subset K$. Fix such a compact set $K$ and choose $K^{\prime} \in \mathcal{K}(\Omega)$ according to $\operatorname{PL}(\Omega, \kappa)$. Next choose $p \in \mathbb{N}$ such that $\left(1+\frac{1}{p}\right) K^{\prime}=: Q$ is in $\mathcal{K}(\Omega)$ and fix $K^{\prime \prime} \in \mathcal{K}(\Omega), K^{\prime \prime} \supset K$ and $l \in \mathbb{N}$. Then let $m:=\max (p, 2 L l)$, where $L \in \mathbb{N}$ is chosen so that by Braun and Meise [5], Prop. 5, there exists $\varphi \in \operatorname{PSH}\left(\mathbb{C}^{n}\right)$ satisfying

$$
-L \omega(z) \leq \varphi(z) \leq|\operatorname{Im} z|-\omega(z) \text { for all } z \in \mathbb{C}^{n} .
$$

Next choose $B=B\left(K^{\prime \prime}\right)$ according to $\operatorname{PL}(\Omega, \kappa)$. Since $\kappa$ is in $S_{\omega}$ there exists $C>0$ such that

$$
2 B \kappa(t) \leq \frac{1}{2 l} \omega(t)+C \text { for all } t>0 .
$$

To show that $V$ satisfies $\widetilde{\mathrm{PL}}\{\Omega, \omega\}$ assume that $u \in \operatorname{PSH}(V)$ satisfies (1) and (2) of $\widetilde{\mathrm{PL}}\{\Omega, \omega\}$. Then we let

$$
v(z):=\frac{m}{m+1}\left(u(z)+\frac{1}{m} \varphi(z)\right), z \in V .
$$

Obviously, $v$ is psh on $V$. From $B_{1}(0) \subset K$ we get

$$
v(z) \leq h_{K}(\operatorname{Im} z)+O(1) \text { and } v(z) \leq h_{K^{\prime \prime}}(\operatorname{Im} z), z \in V .
$$

Since $V$ satisfies $\operatorname{PL}(\Omega, \kappa)$, this implies

$$
v(z) \leq h_{K^{\prime}}(\operatorname{Im} z)+B \kappa(z), z \in V .
$$

By the definition of $v$ and because of our choices, we obtain

$$
\begin{aligned}
u(z) & \leq \frac{m+1}{m} h_{K^{\prime}}(\operatorname{Im} z)+\frac{m+1}{m} B \kappa(z)-\frac{1}{m} \varphi(z) \\
& \leq h_{Q}(\operatorname{Im} z)+\frac{1}{2 l} \omega(z)+C+\frac{1}{2 l} \omega(z) \\
& =h_{Q}(\operatorname{Im} z)+\frac{1}{l} \omega(z)+C
\end{aligned}
$$

which shows that $V$ satisfies $\widetilde{\mathrm{PL}}\{\Omega, \omega\}$.

$(2) \Rightarrow(1)$ : To prove that $V$ satisfies $\operatorname{PL}\{\Omega, \omega\}$, fix $K \in \mathcal{K}(\Omega)$ arbitrarily. Then choose $K^{\prime} \in \mathcal{K}(\Omega)$ according to $\widetilde{\mathrm{PL}}\{\Omega, \omega\}$, let $K^{\prime \prime} \in \mathcal{K}(\Omega)$ and $l \in \mathbb{N}$ be given and choose $m \in \mathbb{N}$ and $C_{l}>0$ according to $\widetilde{\operatorname{PL}}\{\Omega, \omega\}$. If $u \in \operatorname{PSH}(V)$ satisfies (1) and (2) of $\operatorname{PL}\{\Omega, \omega\}$, then it also satisfies (1) and (2) of $\widetilde{\mathrm{PL}}\{\Omega, \omega\}$ for each value of $m$. Hence, by $\widetilde{\mathrm{PL}}\{\Omega, \omega\}$

$$
u(z) \leq h_{K^{\prime}}(\operatorname{Im} z)+\frac{1}{l} \omega(z)+C_{l} \text { for all } z \in V, l \in \mathbb{N} .
$$

This implies that the function $g:[0, \infty[\rightarrow[-\infty, \infty[$,

$$
g(t):=\sup \left\{u(z)-h_{K^{\prime}}(\operatorname{Im} z):|z|=t, z \in V\right\}, t>0,
$$

satisfies $g(t)=o(\omega(t))$ as $t$ tends to infinity. By Braun, Meise and Taylor [6], 1.7, there exists a weight function $\sigma \in S_{\omega}$ such that $g \leq \sigma$. Then the definition of $g$ implies

$$
u(z) \leq h_{K^{\prime}}(\operatorname{Im} z)+\sigma(z) .
$$

Hence $V$ satisfies $\operatorname{PL}\{\Omega, \omega\}$. 
Using Proposition 6.2 we can derive many properties of the condition $\operatorname{PL}\{\Omega, \omega\}$ from the corresponding properties of the condition $\operatorname{PL}(\Omega, \omega)$ that we have already obtained. We note this only in the following three cases.

Theorem 6.3. Let $V$ be a homogeneous variety in $\mathbb{C}^{n}$ and let $\Omega$ be a convex open set in $\mathbb{R}^{n}$. Then the following assertions are equivalent:

(1) $V$ satisfies $\operatorname{PL}\{\Omega, \omega\}$ for some/all weight functions $\omega$.

(2) $V$ satisfies $\operatorname{PL}(\Omega)$.

(3) $V$ satisfies any of the conditions 3.3(4) - 3.3(6).

Proof. (1) $\Rightarrow(2)$ : If $V$ satisfies $\operatorname{PL}\{\Omega, \omega\}$, then $V$ satisfies $\operatorname{PL}(\Omega, \kappa)$ for some $\kappa \in S_{\omega}$, by Proposition 6.2 . Hence $V$ satisfies $\mathrm{PL}(\Omega)$ by Theorem 3.3 .

$(2) \Rightarrow(1)$ : Let $\omega$ be any weight function satisfying $\log =o(\omega)$. Then there exists a weight function $\kappa \in S_{\omega}$ by Braun, Meise and Taylor [6], 1.7. Since $V$ satisfies $\operatorname{PL}(\Omega, \kappa)$ by $(2)$ and Theorem 3.3, we get from Proposition 6.2 that $V$ satisfies $\operatorname{PL}\{\Omega, \omega\}$.

$(3) \Leftrightarrow(2)$ : This holds by Theorem 3.3.

In the same way we deduce the next Theorem from Theorem 4.1 and Proposition 6.2 .

Theorem 6.4. Let $V$ be an algebraic variety in $\mathbb{C}^{n}$ and let $V_{h}$ denote its tangent cone at infinity. If $V$ satisfies $\operatorname{PL}\{\Omega, \omega\}$ for a convex open set $\Omega$ in $\mathbb{R}^{n}$, then $V_{h}$ satisfies $\operatorname{PL}\{\Omega, \omega\}$ and hence $\operatorname{PL}(\Omega)$.

The next Theorem follows from Theorem 5.6 and Proposition 6.2.

Theorem 6.5. Let $V$ be an algebraic variety in $\mathbb{C}^{n}$, let $V_{h}$ denote its tangent cone at infinity and assume that $V_{h} \backslash\{0\}$ is a manifold. Then the following assertions are equivalent:

(1) $V$ satisfies $\operatorname{PL}\left\{\mathbb{R}^{n}, \omega\right\}$.

(2) $V_{h}$ satisfies $\operatorname{PL}\left(\mathbb{R}^{n}\right)$ and $\operatorname{dist}\left(z, V_{h}\right)=o(\omega(z)), z \in V,|z| \rightarrow \infty$.

(3) $V_{h}$ satisfies the strong dimension condition and

$$
\operatorname{dist}\left(z, V_{h}\right)=o(\omega(z)), z \in V,|z| \rightarrow \infty .
$$

Remark. Theorem 6.5 does not hold if we drop the assumption that $V_{h} \backslash\{0\}$ is a manifold outside the origin. This follows from Example 4.9.

\section{REFERENCES}

[1] L.V. Ahlfors, Conformal Invariants; Topics in Geometric Function Theory, McGraw-Hill, 1973. MR 50:10211

[2] K.G. Andersson, Propagation of analyticity of solutions of partial differential equations with constant coefficients, Ark. Mat. 8 (1971), 277-302. MR 45:8986

[3] R.W. Braun, Hörmander's Phragmén-Lindelöf principle and irreducible singularities of codimension 1, Boll. Un. Mat. Ital. 6 -A (1992), 339-348. MR 94b:35012

[4] R.W. Braun, The surjectivity of a constant coefficient homogeneous differential operator on the real analytic functions and the geometry of its symbol, Ann. Inst. Fourier (Grenoble) 45 (1995), 223-249. MR 96e:35025

[5] R.W. Braun and R. Meise, Generalized Fourier expansions for zero-solutions of surjective convolution operators on $\mathcal{D}_{\{w\}}^{\prime}(\mathbb{R})$, Arch. Math. 55 (1990), 55-63. MR 91i:46037

[6] R.W. Braun, R. Meise, and B.A. Taylor, Ultradifferentiable functions and Fourier analysis, Res. Math. 17 (1990), 207-237. MR 91h:46072

[7] R.W. Braun, R. Meise, and D. Vogt, Applications of the projective limit functor to convolution and partial differential equations, in Advances in the Theory of Fréchet Spaces, T. Terzioğlu (Ed.), NATO ASI Series C, Vol. 287 (Kluwer), 1989, 29-46. MR 92b:46119 
[8] R.W. Braun, R. Meise, and D. Vogt, Characterisation of the linear partial differential operators with constant coefficients which are surjective on non-quasianalytic classes of Roumieu type on $\mathbb{R}^{N}$, Math. Nachr. 168 (1994), 19-54. MR 95g:35004

[9] E.M. Chirka, Complex Analytic Sets, Kluwer Academic Publishers, Dordrecht/Boston/London, 1989. MR 92b:32016

[10] D.K. Cohoon, Nonexistence of a continuous linear right inverse for parabolic differential operators, J. Diff. Equ. 6 (1969), 503-511. MR 40:3364

[11] D.K. Cohoon, Nonexistence of a continuous linear right inverse for partial differential operators with constant coefficients, Math. Scand. 29 (1971), 337-342. MR 47:5659

[12] J. Fornaess and R. Narasimhan, The Levi problem on complex spaces with singularities, Math. Ann. 248 (1980), 47-72. MR 81f:32020

[13] L. Hörmander, Linear Partial Differential Operators, Springer, 1963. MR 28:4221

[14] L. Hörmander, Complex Analysis in Several Variables, 2nd Ed. North Holland, 1973. MR 49:9246

[15] L. Hörmander, On the existence of real analytic solutions of partial differential equations with constant coefficients, Invent. Math. 21 (1973), 151-183. MR 49:817

[16] A. Kaneko, On Hartogs type continuation theorem for regular solutions of linear differential equations with constant coefficients, J. Fac. Sci. Tokyo Sect. IA 35 (1988), 1-26. MR 89m:35043

[17] A. Kaneko, Hartogs type extension theorem of real analytic solutions of linear partial differential equations with constant coefficients, in Advances in the Theory of Fréchet Spaces, T. Terzioğlu (Ed.), NATO ASI Series C, Vol. 289 (Kluwer), 1989, 63-72. CMP 91:05

[18] R. Meise, B.A. Taylor, and D. Vogt, Equivalence of slowly decreasing conditions and generalized Fourier expansions, Indiana Univ. J. Math. 36 (1987), 729-750. MR 89c:46058

[19] R. Meise, B.A. Taylor, and D. Vogt, Charactérisation des opérateurs linéaires aux dérivées partielles avec coefficients constants sur $\mathcal{E}\left(\mathbb{R}^{n}\right)$ admettant un inverse à droite qui est linéaire et continu, C. R. Acad. Paris 307 (1988), 239-242. MR 89m:35042

[20] R. Meise, B.A. Taylor, and D. Vogt, Partial differential operators with continuous linear right inverse, in Advances in the Theory of Fréchet Spaces, T. Terzioğlu (Ed.), NATO ASI Series C, Vol. 289 (Kluwer), 1989, pp. 47-72. MR 92b:46122

[21] R. Meise, B.A. Taylor, and D. Vogt, Characterization of the linear partial operators with constant coefficients that admit a continuous linear right inverse, Ann. Inst. Fourier (Grenoble), 40 (1990), 619-655. MR 92e:46083

[22] R. Meise, B.A. Taylor, and D. Vogt, Equivalence of analytic and plurisubharmonic Phragmén-Lindelöf principles on algebraic varieties, Proceedings of Symposia in Pure Mathematics, 52 (1991), 287-308. MR 93a:32023

[23] R. Meise, B.A. Taylor, and D. Vogt, Continuous linear right inverses for partial differential operators on non-quasianalytic classes and on ultradistributions, Math. Nachr. 180 (1996), 213-242. CMP 96:15

[24] R. Meise, B.A. Taylor, and D. Vogt, Indicators of plurisubharmonic functions on algebraic varieties and Kaneko's Phragmén-Lindelöf condition, in Geometrical and Algebraical Aspects in Several Complex Variables, C.A. Berenstein and D.C. Struppa (Eds.), EditEl (1991), pp. 231-250. MR 94d:32018

[25] R. Meise, B.A. Taylor, and D. Vogt, Continuous linear right inverses for partial differential operators with constant coefficients and Phragmén-Lindelöf conditions, in Functional Analysis, K.D. Bierstedt, A. Pietsch, W.M. Ruess, and D. Vogt (Eds.), Lecture Notes in Pure and Applied Math., Vol. 150, Marcel Dekker, 1994, pp. 357-389. MR 94k:35064

[26] R. Meise, B.A. Taylor, and D. Vogt, Extremal plurisubharmonic functions of linear growth on algebraic varieties, Math. Z. 219 (1995), no. 4, 515-537. MR 96j:32019

[27] R. Meise, B.A. Taylor, and D. Vogt, $\omega$-Hyperbolicity of linear partial differential operators with constant coefficients, in Complex Analysis, Harmonic Analysis and Applications (Bordeaux, 1995), Pitman Res. Notes Math. Ser., 347, Longman, Harlow, 1996, 157-182. MR 97h:35027

[28] S. Momm, On the dependence of analytic solutions of partial differential equations on the right-hand side, Trans. Amer. Math. Soc. 345 (1994), 729-752. MR 95a:46036

[29] R. Narasimhan, Introduction to the Theory of Analytic Spaces, Lecture Notes in Math. 25 (1966). MR 36:428 
[30] V.P. Palamodov, A criterion for splitness of differential complexes with constant coefficients, in Geometrical and Algebraical Aspects in Several Complex Variables, C.A. Berenstein and D.C. Struppa (Eds.), EditEl (1991), pp. 265-290. MR 94d:58137

[31] D. Vogt, Some results on continuous linear maps between Fréchet spaces, in Functional Analysis: Surveys and Recent Results II, K.D. Bierstedt and B. Fuchssteiner (Eds), NorthHolland Mathematics Studies, 90 (1984), pp. 349-381. MR 86i:46075

[32] G. Zampieri, An application of the fundamental principle of Ehrenpreis to the existence of global solutions of linear partial differential equations, Boll. U. M. I. 6 (1986), 361-392. MR 88a:35044

Mathematisches Institut, Heinrich-Heine-Universität, Universitätsstrasse 1, 40225 DÜsSEldorf, Germany

E-mail address: meise@cs.uni-duesseldorf.de

Department of Mathematics, University of Michigan, Ann Arbor, Michigan 48109

E-mail address: taylor@math.lsa.umich.edu

Fachbereich Mathematik, Bergische Universität, Gaussstrasse 20, 42097 Wuppertal, Germany

E-mail address: vogt@math.uni-wuppertal.de 